\title{
Introducing two New Sieves for Factorization Natural Odd Numbers
}

\author{
Ramazanali Maleki Chorei \\ Department of Mathematics, Roudbar Branch, Islamic Azad University, Roudbar, Iran \\ Corresponding author: Dr.Maleki@iauroudbar.ac.ir
}

\begin{abstract}
For each non-prime odd number as $F=p q$, if we consider $m / n$ as an approximation for $q / p$ and choose $k=m n$, then by proving some lemmas and theorems, we can compute the values of $m$ and $n$. Finally, by using Fermat's factorization method for $F$ and $4 k F$ as difference of two non-consecutive natural numbers, we should be able to find the values of $p$ and $q$. Then we introduce two new and powerful sieves for separating composite numbers from prime numbers.
\end{abstract}

Keywords: Prime numbers, lemmas and theorems, Fermat's factorization method, sieve

\section{Introduction}

There are many features for identification of prime numbers from non-prime numbers. In this paper, we attempt to identify very important properties about non-prime odd numbers by proving some theorems and lemmas. In 1643, Fermat a French mathematician described a method for factorization of big odd numbers by a letter to Marin Mersenne. In composite numbers as $F=p q$, by propose $k_{r}=q / p \approx m / n$ and $K=m n$,we introduce two new sieves for factorization of non-prime odd numbers by developing Fermat's factorization method. The first sieve ( $\alpha-s$ sieve ) based on the relation $(\lfloor\sqrt{F}\rfloor+\theta)^{2}-$ $F=\left(\frac{p-q}{2}\right)^{2}$ and the second sieve $(\beta-s$ sieve $)$ based on the relation $(\lfloor\sqrt{4 k F}\rfloor+\theta)^{2}-4 k F=(m p-$ $n q)^{2}$ ( $\theta$ is a natural number). We show these sieves are very convenient for big numbers, because we don't use long calculation within process of them. 


\section{Development of Fermat's factorization method}

We know each non-prime odd number as $F=p q(3 \leq p<q)$, can be written as a difference of squares of two nonconsecutive natural numbers as following:

$\left\{\begin{array}{c}p=2 m+1 \\ q=2 n+1\end{array} \Rightarrow F=p q=(m+n+1)^{2}-(m-n)^{2}\right.$

It is clear that by assuming $k_{r}=\frac{q}{p}$, we have $F=k_{r} p^{2}$.

Therefore, for each natural number bigger than 1 as $k$, we can write $4 k F$ as difference of squares of two even numbers or odd numbers, in which $F$ is a non-prime odd number:

$4 k F=4 k(p q)=(2 k p)(2 q)=(q+k p)^{2}-(q-k p)^{2}$

If we assume $k=m n$, in this case, we can write:

$4 k F=4 m n p q=(m p+n q)^{2}-(m p-n p)^{2}$

Definition: In this paper, the expressions $(\lfloor\sqrt{4 k F}\rfloor+\theta)^{2}-4 k F$ and $(\lfloor\sqrt{F}\rfloor+\theta)^{2}-F$ are shown by $\beta(k, \theta)$ and $\alpha(\theta)$ respectively.

If $F$ is a square number, this means that it's a non-prime number and if $F$ isn't a square number, and $\alpha(\theta)$ is a square, then according to the identity $F=p q=\left(\frac{p+q}{2}\right)^{2}-\left(\frac{p-q}{2}\right)^{2}$ and

definition of $\alpha(\theta)$, we have:

$\alpha(\theta)=(\lfloor\sqrt{F}\rfloor+\theta)^{2}-F \Rightarrow\left\{\begin{array}{l}\frac{p+q}{2}=\lfloor\sqrt{F}\rfloor+\theta \\ \frac{p-q}{2}=\sqrt{\alpha(\theta)}\end{array} \Rightarrow\left\{\begin{array}{l}p=(\lfloor\sqrt{F}\rfloor+\theta)-\sqrt{\alpha(\theta)} \\ q=(\lfloor\sqrt{F}\rfloor+\theta)+\sqrt{\alpha(\theta)}\end{array}\right.\right.$

If $k F$ is a square number and assume $k_{r}=\frac{q}{p}$, it means that:

$F=k_{r} p^{2} \Rightarrow k F=k k_{r} p^{2} \Rightarrow k k_{r}=t^{2}$

Since $t$ and $k$ are natural numbers, so $F$ will be divided on $k_{r}$. This means that $F$ is a non-prime number. If $k F$ isn't a square number and $\beta(k, \theta)$ is a square number, then we will have:

$$
\begin{aligned}
& 4 k F=(q+k p)^{2}-(q-k p)^{2}=(\lfloor\sqrt{4 k F}\rfloor+\theta)^{2}-\beta(k, \theta) \Rightarrow\left\{\begin{array}{l}
q+k p=\lfloor\sqrt{4 k F}\rfloor+\theta \\
|q-k p|=\sqrt{\beta(k, \theta)}
\end{array}\right. \\
& \Rightarrow p=\frac{(\lfloor\sqrt{4 k F}\rfloor+\theta) \mp \sqrt{\beta(k, \theta)}}{2 k}, q=\frac{(\lfloor\sqrt{4 k F}\rfloor+\theta) \pm \sqrt{\beta(k, \theta)}}{2}
\end{aligned}
$$


By assuming $k=m n$, we will have:

$p=\frac{(\lfloor\sqrt{4 k F}\rfloor+\theta) \mp \sqrt{\beta(k, \theta)}}{2 m}, q=\frac{(\lfloor\sqrt{4 k F}\rfloor+\theta) \pm \sqrt{\beta(k, \theta)}}{2 n}$

When $F$ is a non-prime number, both $p$ and $q$ are natural numbers bigger than 1.

Lemma 1: Whenever $k$ is a natural even number such that $x>\frac{(K-2)^{2}}{8}$ and $x$ is a natural number, in this case, we will have:

$\left\lfloor\sqrt{x^{2}+k x}\right\rfloor=x+\frac{k-2}{2}$

Proof: From the basic algebra, we have:

$\left(x+\frac{k}{2}-1\right)^{2}<x^{2}+k x<\left(x+\frac{k}{2}\right)^{2} \Rightarrow x+\frac{k}{2}-1<\sqrt{x^{2}+k x}<x+k$.

If $x+\frac{K}{2}-1$ isn't the biggest integer number smaller than $\sqrt{x^{2}+k x}$, we should have at least:

$\left(x+\frac{K}{2}-1\right)+1<\sqrt{x^{2}+k x} \Rightarrow x^{2}+k x+\frac{K^{2}}{4}<x^{2}+k x$

Since $\frac{K^{2}}{4}>0$, therefore the inequality (1) isn't correct. So $x+\frac{K}{2}-1$ is the biggest integer number smaller than $\sqrt{x^{2}+k x}$ and based on the bracket function definition, we will have

$\left\lfloor\sqrt{x^{2}+k x}\right\rfloor=x+\frac{k-2}{2}$

Because $\left(x+\frac{K}{2}-1\right)^{2}<x^{2}+k x$, so we will have $x>\frac{(K-2)^{2}}{8}$

Lemma 2: Suppose $x$ is a natural number and $k$ is a natural even number such that $x>\frac{(K+2)^{2}}{8}$. Then, we will have:

$\left\lfloor\sqrt{x^{2}-k x}\right\rfloor=x-\frac{k+2}{2}$

Proof: From basic algebra, we have:

$\left(x-\frac{k+2}{2}\right)^{2}<x^{2}-k x<\left(x-\frac{k}{2}\right)^{2} \Rightarrow x-\frac{k+2}{2}<\sqrt{x^{2}-k x}<x-\frac{k}{2}$.

If $x-\frac{k+2}{2}$ isn't the biggest integer component smaller than $\sqrt{x^{2}+k x}$, then we should have: 


$$
\left(x-\frac{k+2}{2}\right)+1<\sqrt{x^{2}-k x} \Rightarrow\left(x^{2}-k x\right)+\frac{k^{2}}{4}<x^{2}-k x
$$

Because $\frac{k^{2}}{4}>0$, so the inequality (1) is incorrect and $x-\frac{k+2}{2}$ is the biggest integer number smaller than $\sqrt{x^{2}-k x}$ and from bracket function definition, we will have:

$\left\lfloor\sqrt{x^{2}-k x}\right\rfloor=x-\frac{k+2}{2}$

And so:

$\left(x-\frac{k+2}{2}\right)^{2}<x^{2}-k x \Rightarrow x>\frac{(k+2)^{2}}{8}$.

In this paper, the approximate value of a number as $x$ will be shown in the form of $\sim(x)$ or $\tilde{x}$.

Theorem 1: For each natural odd number as $F=p q,(1 \leq p<q)$ if we assume $\tilde{k}_{r}=\sim(q / p)=m / n$ and $\left|\tilde{k}_{r}-k_{r}\right|=0 \cdot \overline{a_{1} \ldots a_{s}}$, by choosing $k=m n$, the result of $\beta(k, 1)$ will be square when $\overline{a_{1} \ldots \overline{a_{s}}} p^{2}<$ $\sqrt{4 F \times 10^{s}} \pm 1$.

Proof: If $\tilde{k}_{r}>k_{r}$, we can write:

$\widetilde{K}_{r}=\frac{m}{n}=\frac{q}{p}+0 . \overline{a_{1} \ldots a_{s}}=\frac{10^{S} q+\overline{a_{1} \ldots a_{s}} p}{10^{S} p}$

If we choose $m=10^{S} q+\overline{a_{1} \ldots a_{s}} p$ and $n=10^{S} p$, then we will have:

$k=m n=10^{2 S} F+\overline{a_{1} \ldots a_{s}} p^{2} \times 10^{S} \Rightarrow 4 k F=\left(2 \times 10^{S} F\right)^{2}+\left(2 p^{2} \overline{a_{1} \ldots \overline{a_{s}}}\right)\left(2 \times 10^{S} F\right)$

Now, by assuming $\overline{a_{1} \ldots a_{s}}\left(2 p^{2}\right)=k^{\prime}$ and $x=2 \times 10^{S} F$, we arrive at $4 k F=x^{2}+k^{\prime} x$. Since $k^{\prime}$ is even, so by lemma (1), we get

$\lfloor\sqrt{4 k F}\rfloor=\left\lfloor\sqrt{x^{2}+k^{\prime} x}\right\rfloor=x+\frac{k^{\prime}-2}{2}=2 \times 10^{S} F+\overline{a_{1} \ldots a_{s}} p^{2}-1 \Rightarrow$

$\beta(k, 1)=(\lfloor\sqrt{4 k F}\rfloor+1)^{2}-4 k F=\left(2 \times 10^{S} F+\overline{a_{1} \ldots a_{s}} p^{2}\right)^{2}-4 k F$,

and therefore, according to the relation (1), we will obtain $\beta(k, 1)=\left(\overline{a_{1} \ldots a_{s}} P^{2}\right)^{2}$. Consequently, based on the lemma (1) and by assuming $x>\frac{\left(k^{\prime}-2\right)^{2}}{8}$, we should have:

$k^{\prime}<\sqrt{8 x}+2 \Rightarrow \overline{a_{1} \ldots a_{s}} . P^{2}<\sqrt{4 F \times 10^{S}}+1$

In the case that $\tilde{k}_{r}<k_{r}$, the proof is similar to the above and we should have:

$\overline{a_{1} \ldots a_{s}} P^{2}<\sqrt{4 F \times 10^{S}}-1$. 
Note1: For each natural odd number, when $p=1$, then we have $\beta(k, 1)=\left(\overline{a_{1} \ldots a_{s}}\right)^{2}$.

Whenever $\left|\tilde{k}_{r}-k_{r}\right|=10^{-s}$, then we have $\beta(k, 1)=1$.

Theorem 2: In a non-prime odd number as $F=p q(1 \leq p<q)$, if $p>\sqrt{F}+1-\sqrt{(\sqrt{F}+1)^{2}-F}$, then the result of $\alpha(1)$ will be square and we will have:

$$
\alpha(1)=(\lfloor\sqrt{F}\rfloor+1)^{2}-F=\left(\frac{\delta}{2}\right)^{2}=\left(\frac{q-p}{2}\right)^{2}
$$

Proof: Since $\delta=q-p$ is even, then from lemma (2) we have:

$\lfloor\sqrt{F}\rfloor=\lfloor\sqrt{p q}\rfloor=\lfloor\sqrt{p(p+\delta)}\rfloor=\left\lfloor\sqrt{p^{2}+\delta . P}\right\rfloor=p+\frac{\delta-1}{2}=\frac{q+p}{2}-1$

$\Rightarrow \alpha(1)=\left(\frac{p+q}{2}\right)^{2}-p q=\left(\frac{q-p}{2}\right)^{2}=\left(\frac{\delta}{2}\right)^{2}$

In this case, according to lemma (1), we have:

$\delta=q-p<\sqrt{8 p}+2=2 \sqrt{2 p}+2 \Rightarrow \frac{F}{p}-p<2 \sqrt{2 p}+2 \Rightarrow F<(p+\sqrt{2 p})^{2}$

$\Rightarrow p+\sqrt{2 p}-\sqrt{F}>0 \Rightarrow P>\sqrt{F}+1-\sqrt{(\sqrt{F}+1)^{2}-F}$.

Lemma 3: If $k$ is a natural odd number and $x$ is a natural number such that $x>\left(\frac{k-1}{2}\right)^{2}$, then we will have:

$$
\left\lfloor\sqrt{x^{2}+k x}\right\rfloor=x+\frac{k-1}{2}
$$

Proof: From the basic algebra, we will have:

$$
\left(x+\frac{k-1}{2}\right)^{2}<x^{2}+k x<\left(x+\frac{k}{2}\right)^{2} \Rightarrow x+\frac{k-1}{2}<\sqrt{x^{2}+k x}<x+\frac{k}{2} .
$$

If $x+\frac{k-1}{2}$ is not the biggest integer number smaller than $\sqrt{x^{2}+k x}$, then, we should have at least:

$$
\left(x+\frac{k-1}{2}\right)+1<\sqrt{x^{2}+k x} \Rightarrow\left(x+\frac{k+1}{2}\right)^{2}<x^{2}+k x \Rightarrow\left(x^{2}+k x\right)+x+\left(\frac{k+1}{2}\right)^{2}<x^{2}+k x
$$

Because $x+\left(\frac{k+1}{2}\right)^{2}>0$, so the inequality (1) is not correct and therefore this means that $x+\frac{k-1}{2}$ is the biggest integer number smaller than $\sqrt{x^{2}+k x}$. Then, according to definition of the bracket function, we should have $\left[\sqrt{x^{2}+k x}\right]=x+\frac{k-1}{2}$. But $\left(x+\frac{k-1}{2}\right)^{2}<x^{2}+k x$, so we should have 
$x>\left(\frac{k-1}{2}\right)^{2}$.

Theorem3: In a natural odd number as $F=p q,(1 \leq p<q)$, by assuming $k_{r}=q / p$ and $\tilde{k}_{r}=$ $\sim(q / p)=m / n$, we will have $\beta(k, 1)=(m p-n q)^{2}$.

In the case that $\tilde{k}_{r}>k_{r}$,we should have $|m p-n q|=0 . \overline{a_{1} \ldots a_{s}} n p<2 \sqrt{n q}+1$,

and in the case that $\tilde{k}_{r}<k_{r}$, we should have $|m p-n q|=0 . \overline{a_{1} \ldots a_{s}} n p<2 \sqrt{m p}+1$.

Proof: By assuming $k=m n$,we have $\lfloor\sqrt{4 k F}\rfloor=\lfloor\sqrt{4 m n p q}\rfloor=\lfloor\sqrt{P Q}\rfloor$ which $P=2 m p$ and $Q=2 n q$.

If $\tilde{k}_{r}>k_{r}: \frac{m}{n}>\frac{q}{p} \Rightarrow \tilde{k}_{r}-k_{r}=0 . \overline{a_{1} \ldots \bar{a}_{s}}=\frac{m}{n}-\frac{q}{p}=\frac{m p-n q}{n p}$.

According to lemma (1), we will have:

$\delta=P-Q=2 m p-2 n q \Rightarrow\lfloor\sqrt{4 k F}\rfloor=\lfloor\sqrt{P Q}\rfloor=\lfloor\sqrt{Q(Q+\delta)}\rfloor=Q+\frac{\delta-2}{2}$

$=\frac{P+Q}{2}-1=(m p+n q)-1 \Rightarrow \beta(k, 1)=(m p+n q)^{2}-4 m n p q=(m p-n q)^{2}$

Based on lemma (1), we have:

$\delta=P-Q<\sqrt{8 Q}+2 \Rightarrow m p-n q<2 \sqrt{n q}+1$

and according to the relation (1), we can write $m p-n q=0 . \overline{a_{1} \ldots a_{s}} n p<2 \sqrt{n q}+1$.

If $\tilde{k}_{r}<k_{r}: \frac{m}{n}<\frac{q}{p} \Rightarrow k_{r}-\tilde{k}_{r}=0 . \overline{a_{1} \ldots \bar{a}_{s}}=\frac{q}{p}-\frac{m}{n}=\frac{n q-m p}{n p}$.

According to lemma (1), we have:

$\delta=Q-P=2 n q-2 m p \Rightarrow\lfloor\sqrt{4 k F}\rfloor=\lfloor\sqrt{4 m n p q}\rfloor=\lfloor\sqrt{P Q}\rfloor=\lfloor\sqrt{P(P+\delta)}\rfloor=\left\lfloor\sqrt{P^{2}+P \delta}\right\rfloor$
$=P+\frac{\delta-2}{2}=\frac{P+Q}{2}-1=n q+m p-1 \Rightarrow \beta(k, 1)=(n q+m p)^{2}-4 m n p q=(m p-n q)^{2}$.

According to lemma (1), we should have:

$\delta=Q-P<\sqrt{8 P}+2 \Rightarrow n q-m p<2 \sqrt{m p}+1$

Based on the relation (2), we will have: $n q-m p=0 . \overline{a_{1} \ldots \bar{a}_{s}} n p<2 \sqrt{m p}+1$. 
Theorem 4: For each natural odd number as $F=p q$, $(1 \leq p<q)$, the value of $\beta(k, 1)$ will be square when $k$ lies in interval $\left(\frac{(\sqrt{q}+1)^{2}}{P}, \frac{(\sqrt{q}-1)^{2}}{P}\right)$ except in a case that $k=k_{r}$.

Proof (1): If $k>\frac{q}{p}$, in this case $2 k p>2 q$ and because the result of $\delta=2 k p-2 q$ is even, then from lemma (1), we will have:

$\lfloor\sqrt{4 k F}\rfloor=\lfloor\sqrt{(2 k p)(2 q)}\rfloor=\lfloor\sqrt{2 q(2 q+\delta)}\rfloor=\left\lfloor\sqrt{(2 q)^{2}+2 q \delta}\right\rfloor=2 q+\frac{\delta-2}{2}=q+k p-1$

$\Rightarrow \beta(k, 1)=(q+k p)^{2}-4 k p q=(q-k p)^{2}$.

According to lemma (1), we should have:

$\delta=2 k p-2 q<\sqrt{8(2 q)}+2 \Rightarrow k p-q-1<2 \sqrt{q} \Rightarrow k p<(\sqrt{q}+1)^{2} \Rightarrow k<\frac{(\sqrt{q}+1)^{2}}{p}$

If $k<\frac{q}{p}$, in this case $2 k p<2 q$ and because the result of $\delta=2 q-2 k p$ is even, then from lemma (1), we will get:

$\lfloor\sqrt{4 k F}\rfloor=\lfloor\sqrt{(2 k p)(2 q)}\rfloor=\lfloor\sqrt{(2 k p)(2 k p+\delta)}\rfloor=\left\lfloor\sqrt{(2 k p)^{2}+2 k p \delta}\right\rfloor=2 k p+\frac{\delta-2}{2}$

$=q+k p-1 \Rightarrow \beta(k, 1)=(q+k p)^{2}-4 k p q=(q-k p)^{2}$.

Based on the lemma (1), we should have:

$\delta=2 q-2 k p<\sqrt{8(2 k p)}+2 \Rightarrow q-k p<2 \sqrt{k p}+1 \Rightarrow q<(\sqrt{k p}+1)^{2} \Rightarrow k>\frac{(\sqrt{q}-1)^{2}}{p}$.

Now, from (1) and (2), we will have $\frac{(\sqrt{q}-1)^{2}}{p}<k<\frac{(\sqrt{q}+1)^{2}}{p}$, thus if $k=k_{r}$, we can write:

$k=k_{r}=\frac{q}{p} \Rightarrow 4 k F=4 q^{2} \Rightarrow \beta(k, 1)=(2 q+1)^{2}-4 q^{2}=4 q+1 \neq|k p-q|=0$

so, we can conclude $k \neq k_{r}$.

Proof (2): According to theorem(3), when we assume $k=\tilde{k}_{r}=m$ and $n=1$, we will have:

$$
\begin{aligned}
& \left\{\begin{array} { l } 
{ \tilde { k } _ { r } > k _ { r } \Rightarrow k p - q < 2 \sqrt { q } + 1 } \\
{ \tilde { k } _ { r } < k _ { r } \Rightarrow q - k p < 2 \sqrt { k p } + 1 }
\end{array} \Rightarrow \left\{\begin{array}{l}
k<\frac{(\sqrt{q}+1)^{2}}{p} \\
k>\frac{(\sqrt{q}-1)^{2}}{p}
\end{array} \Rightarrow \frac{(\sqrt{q}-1)^{2}}{p}<k<\frac{(\sqrt{q}+1)^{2}}{p}\right.\right. \\
& \text { or }\left(\frac{\sqrt{F}-\sqrt{P}}{p}\right)^{2}<k<\left(\frac{\sqrt{F}+\sqrt{P}}{P}\right)^{2}
\end{aligned}
$$


If we propose $k_{\min }=\frac{(\sqrt{q}-1)^{2}}{p}$ and $k_{\max }=\frac{(\sqrt{q}+1)^{2}}{p}$ and shown the difference of $K_{\min }$ and $K_{\max }$ by $\delta$ then for every integer number which is lied in that interval, the result of $\beta(k, 1)$ will be square. Therefore, we have $\delta=k_{\text {max }}-k_{\min }=\frac{4 \sqrt{q}}{p}$.

We will have the Maximum value of $\delta$ when $P=1$. In this case, we have $\delta_{\max }=4 \sqrt{F}$.

If the number of natural numbers located in interval $\left(k_{\min }, k_{\max }\right)$ are demonstrated by $N$, so we will have $N=\left\lfloor k_{\text {max }}\right\rfloor-\left\lfloor k_{\text {min }}\right\rfloor$.

If $k=k_{r}$ and it is located in interval $\left(K_{\text {min }}, K_{\text {max }}\right)$, then we will have $N=\left\lfloor K_{\text {max }}\right\rfloor-\left\lfloor K_{\text {min }}\right\rfloor-1$.

When the value of $\delta$ is maximum, then the value of $N$ will be maximum too.

$\left\{\begin{array}{l}P=1 \\ q=F\end{array} \Rightarrow\left\{\begin{array}{l}\left\lfloor k_{\text {min }}\right\rfloor=\left\lfloor(\sqrt{F}-1)^{2}\right\rfloor \\ \left\lfloor k_{\text {max }}\right\rfloor=\left\lfloor(\sqrt{F}+1)^{2}\right\rfloor\end{array} \Rightarrow N_{\text {max }}=\left\lfloor k_{\text {max }}\right\rfloor-\left\lfloor k_{\text {min }}\right\rfloor-1\right.\right.$

$=\lfloor F+2 \sqrt{F}+1\rfloor-\lfloor F-2 \sqrt{F}+1\rfloor-1=(F+1)+\lfloor 2 \sqrt{F}\rfloor-(F+1)-\lfloor-2 \sqrt{F}\rfloor-1$

$=\lfloor 2 \sqrt{F}\rfloor-(-\lfloor 2 \sqrt{F}\rfloor-1)-1=\lfloor 4 \sqrt{F}\rfloor$.

Example1: Find the value of $N$ in $F=17 \times 43=6851$.

Answer: $p=17, q=43 \Rightarrow 21<k \leq 26 \Rightarrow N=26-21=5$.

Then we can see that:

$k=22 \Rightarrow \beta(22,1)=5^{2}, k=23 \Rightarrow \beta(23,1)=12^{2}, k=24 \Rightarrow \beta(24,1)=5^{2}$,

$k=25 \Rightarrow \beta(25,1)=44^{2}, k=26 \Rightarrow \beta(26,1)=39^{2}$.

Theorem 5: For odd natural numbers as $F=p q$, by propose $k_{r}=\frac{q}{p} \simeq \frac{m}{n},(m, n)=1$ and choose $k=$ $r^{2} m n$, when $r$ is a natural number, then the value of $\beta(k, 1)$ will be square.

Proof: For proof in the first case, we propose $\frac{m}{n}>\frac{q}{p}$

Therefore $\frac{r m}{r n}>\frac{q}{p}=>\quad r m p>r n p=>\quad\lfloor\sqrt{4 k F}\rfloor=\lfloor\sqrt{(2 r m p)(2 r n q)}\rfloor=\sqrt{P Q}$

If $P=2 r m p, Q=2 r n q$, then we have $\delta=P-Q=2 r m p-2 r n q>0$.

Based on lemma (1), we should have: 
$\lfloor\sqrt{P Q}\rfloor=\lfloor\sqrt{Q(Q+\delta)}\rfloor=Q+\frac{\delta-2}{2}=\frac{P+Q}{2}-1=r m p-r n q-1=>$

$\beta(k, 1)=(\mid \sqrt{4 k F}\rfloor+1)^{2}-4 k F=(r m p+r n q)^{2}-4 r^{2} m n p q=(r m p-r n q)^{2}$.

Therefore we should have $r m p-r n q<2 \sqrt{r n q}+1$.

In the case $\frac{m}{n}<\frac{q}{p}$, the proof is similar as above.

Theorem 6: For each non-prime odd number as $F=p q(1 \leq p<q)$, if we assume

$\theta=\left[(\sqrt{k p}-\sqrt{q})^{2}\right\rfloor+1$ and $k F$ doesn't be square, then the value of $\beta(k, \theta)$ will be square so that

$\frac{(\sqrt{q}+\sqrt{\theta-1})^{2}}{p} \leq k<\frac{(\sqrt{q}+\sqrt{\theta})^{2}}{p}$ and $\frac{(\sqrt{q}-\sqrt{\theta})^{2}}{p}<k \leq \frac{(\sqrt{q}-\sqrt{\theta-1})^{2}}{p}$

Proof: In the identity $4 k F=4 k(p q)=(q+k p)^{2}-(q-k p)^{2}$,since $k F$ can not be square, thus we have $\theta=\lfloor k p+q-\sqrt{4 k p q}\rfloor+1=k p+q+\lfloor-\sqrt{4 k p q}\rfloor+1=k p+q+(-\lfloor\sqrt{4 k p q}\rfloor-1)+1$

$=k p+q-\lfloor\sqrt{4 k p q}\rfloor \Rightarrow \beta(k, \theta)=(k p+q)^{2}-4 k p q=(k p-q)^{2}$.

At that rate for any $k$ value, we can write:

$$
\begin{aligned}
& \theta=\left|(\sqrt{k p}-\sqrt{q})^{2}\right|+1=>\theta-1 \leq(\sqrt{k p}-\sqrt{q})^{2}<\theta=>\sqrt{\theta-1} \leq|\sqrt{k p}-\sqrt{q}|<\sqrt{\theta}=> \\
& \frac{(\sqrt{q}+\sqrt{\theta-1})^{2}}{p} \leq k<\frac{(\sqrt{q}+\sqrt{\theta})^{2}}{p}, \quad \frac{(\sqrt{q}-\sqrt{\theta})^{2}}{p}<k \leq \frac{(\sqrt{q}-\sqrt{\theta-1})^{2}}{p}
\end{aligned}
$$

In the sequel we have:

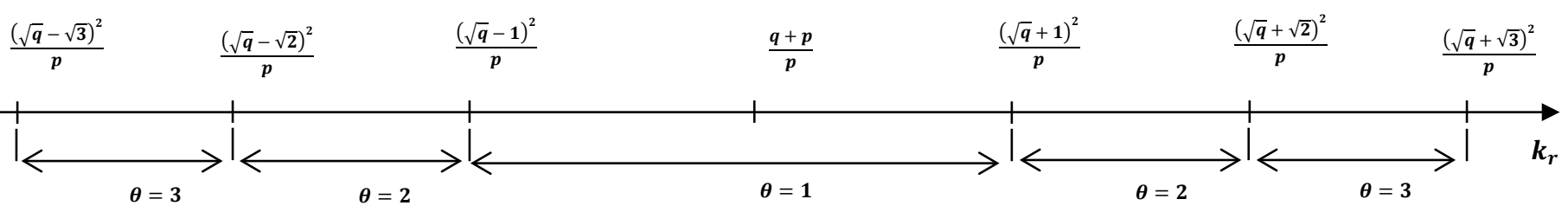

For example when $F=89 \times 911=81,079$ and $\theta=7$, we can calculate $k$ values as bellow:

$\frac{(\sqrt{q}+\sqrt{\theta-1})^{2}}{p} \leq k<\frac{(\sqrt{q}+\sqrt{\theta})^{2}}{p}=>\frac{(\sqrt{911}+\sqrt{6})^{2}}{89} \leq k<\frac{(\sqrt{911}+\sqrt{7})^{2}}{89}=>$

$11 / 9 \leq k<12 / 1=>k=12=>\beta(k, \theta)=\beta(12,7)=(1972+7)^{2}-3,891,792=157^{2}$ 
We can observe when $\theta=6$, we can't find any value for $k$.

Note 2: For any value of $k$ as a natural number, exist a nonnegative integer number as $\theta$ $\left(\theta=\left\lfloor(\sqrt{k p}-\sqrt{q})^{2}\right\rfloor+1\right)$, so that $\beta(k, \theta)$ should be square. Whiles for any value of $\theta$, may don't be exist a value for $k$.

Generalized form of theorem 6: For each non-prime odd number as $F=p q(1 \leq p<q)$, by assuming $m>n$ ( $m, n$ are natural numbers), if we choose $\theta=\left\lfloor(\sqrt{m p}-\sqrt{n q})^{2}\right\rfloor+1$ and mnpq doesn't be square, then the value of $\beta(k, \theta)$ will be square.

Proof: According to the identity $4 k F=4 m n p q=(m p+n q)^{2}-(m p-n p)^{2}$ and if $4 m n p q$ doesn't be square, so we should have:

$\theta=\lfloor m p+n q-2 \sqrt{m n p q}\rfloor+1=m p+n q+\lfloor-\sqrt{4 m n p q}\rfloor+1$

$=m p+n q+(-\lfloor\sqrt{4 m n p q}\rfloor-1)+1=m p+n q-\lfloor\sqrt{4 m n p q}\rfloor$

$\Rightarrow \beta(k, \theta)=(\lfloor\sqrt{4 m n p q}\rfloor+\theta)^{2}-4 m n p q=(m p-n q)^{2}$.

For example, when $F=17 \times 23=391$ and propose $m=5$ and $n=2$, then we should have:

$\theta=\left\lfloor(\sqrt{m p}-\sqrt{n q})^{2}\right\rfloor+1=\left\lfloor(\sqrt{5 \times 17}-\sqrt{2 \times 23})^{2}\right\rfloor+1=6$ and $k=m n=10 \Rightarrow$

$\beta(k, \theta)=\beta(10,6)=(125+6)^{2}-15640=1521=39^{2}$

Generalized form of theorem 4: For each non-prime natural numbers as $F=p q(1 \leq p<q)$, if we have $\tilde{k}_{r}=\sim(q / p)=m / n$ and $k=m n$, then the value of $\beta(k, 1)$ would be square whenever:

$\frac{(\sqrt{n q}-1)^{2}}{p}<m<\frac{(\sqrt{n q}+1)^{2}}{p}$

Proof: From theorem (3), we have:

$$
\begin{aligned}
& \left\{\begin{array}{l}
\tilde{k}_{r}>k_{r} \Rightarrow m p-n q<2 \sqrt{n q}+1 \Rightarrow m p<(\sqrt{n q}+1)^{2} \Rightarrow m<\frac{(\sqrt{n q}+1)^{2}}{p} \\
\tilde{k}_{r}<k_{r} \Rightarrow n q-m p<2 \sqrt{m p}+1 \Rightarrow n q<(\sqrt{m p}+1)^{2} \Rightarrow m>\frac{(\sqrt{n q}-1)^{2}}{p}
\end{array}\right. \\
& \Rightarrow \frac{(\sqrt{n q}-1)^{2}}{p}<m<\frac{(\sqrt{n q}+1)^{2}}{p} .
\end{aligned}
$$


According to $q=\sqrt{k_{r} F}$ and $P=\sqrt{\frac{F}{k_{r}}}$ as well as assuming $M_{\max }=\frac{(\sqrt{n q}+1)^{2}}{p}$ and $M_{\min }=\frac{(\sqrt{n q}-1)^{2}}{p}$, if difference of $M_{\min }$ and $M_{\max }$ will be shown by $\delta$, so we will get:

$\delta=M_{\max }-M_{\min } \Rightarrow \delta=\frac{4 \sqrt{n q}}{p}=4 \sqrt{\frac{n F}{P^{3}}}=4 \sqrt[4]{\frac{k_{r}^{3} n^{2}}{F}}$.

If we propose $\tilde{k}_{r}=\frac{m}{n}=a \cdot \overline{b_{1} b_{2} \ldots b_{s}}=\frac{\overline{a b_{1} \ldots b_{s}}}{10^{s}}$ then by choosing $n=10^{s}$ and $=\overline{a b_{1} \ldots b_{s}}$, we should have:

$$
\begin{aligned}
& \frac{\left(\sqrt{10^{s} q}-1\right)^{2}}{p}<m<\frac{\left(\sqrt{10^{s} q}+1\right)^{2}}{p} \text { or }\left(\frac{\sqrt{10^{S} F}-\sqrt{p}}{p}\right)^{2}<m<\left(\frac{\sqrt{10^{S} F}+\sqrt{p}}{p}\right)^{2} \\
& \Rightarrow \delta=\frac{4 \sqrt{10^{s} q}}{p}=4 \sqrt{\frac{10^{s} F}{p^{3}}}=4 \sqrt[4]{\frac{k_{r}^{3} \times 10^{2 s}}{F}}
\end{aligned}
$$

If we show the number of natural numbers that is located in interval $\left(M_{\min }, M_{\max }\right)$ by $N$, so for any $k_{r}$ we should have:

$\left\lfloor M_{\text {max }}\right\rfloor-\left\lfloor M_{\text {min }}\right\rfloor=N \Rightarrow N-1<\delta<N+1$ or $\delta-1<N<\delta+1$

In other way $\lfloor\delta\rfloor=N$ or $\lfloor\delta\rfloor=N-1$, therefore for each value of $s$ and $N$, we can conclude:

$\sqrt[3]{\left(\frac{N-1}{4}\right)^{4} \frac{F}{10^{2 S}}}<k_{r}<\sqrt[3]{\left(\frac{N+1}{4}\right)^{4} \frac{F}{10^{2 S}}}$

For any value of $S$, we define $k_{(s): i}$ as below

$k_{(s): i}=\sqrt[3]{\left(\frac{i}{4}\right)^{4} \frac{F}{10^{2 s}}} \Rightarrow\left\{\begin{array}{l}N=1=>1 \leq k_{r}<k_{(s): 2} \\ N=2=>\quad k_{(s): 1} \leq k_{r}<k_{(s): 3} \\ \ldots \ldots \ldots \ldots \ldots \ldots \ldots \ldots \ldots \ldots \ldots \ldots \ldots \ldots \ldots \ldots \ldots \ldots \ldots \ldots \ldots \\ N=i+1=>\quad k_{(s): i} \leq k_{r}<k_{(s):(i+2)}\end{array}\right.$

Then for any $k_{(s): i}$ we have:

$\left\{\begin{array}{l}\delta=\sqrt[4]{\frac{k_{r}^{3} \times 10^{2 s}}{F}} \\ k_{(s): 1}=\sqrt[3]{\left(\frac{i}{4}\right)^{4} \frac{F}{10^{2 s}}}\end{array}=>\delta=i=>N=i\right.$

Since we have $k_{(s): i} \leq k_{(s-1): i}$, therefore we should have: 
$\sqrt[3]{\left(\frac{i}{4}\right)^{4} \frac{F}{10^{2 s}}} \leq \sqrt[3]{\left(\frac{1}{4}\right)^{4} \frac{F}{10^{2(s-1)}}}=>i<\sqrt{10} \Rightarrow i_{\max }=\lfloor\sqrt{10}\rfloor=3$

We can calculate $s_{\max }$ for F as below:

$\sqrt[3]{\left(\frac{1}{4}\right)^{4} \frac{F}{10^{2 s}}}=1=>s=\log \frac{\sqrt{F}}{16}=>s_{\max }=\left\lceil\log \frac{\sqrt{F}}{16}\right\rceil$

If we propose i as a natural number from zero to $s_{\max }$, then define s value as $s=s_{\max }-i$.

For any s we have:

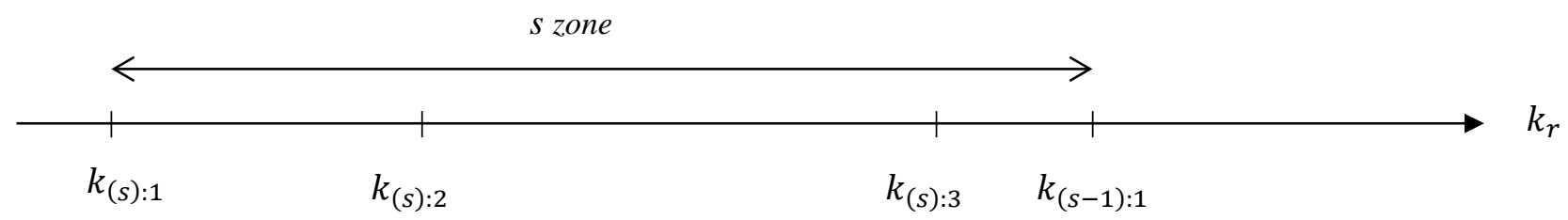

For any s we have:

$k_{(s): 1}=\sqrt[3]{\left(\frac{1}{4}\right)^{4} \frac{F}{10^{2\left(s_{\max }-i\right)}}}<\sqrt[3]{\left(\frac{1}{4}\right)^{4} \frac{F}{10^{2(s-i)}}}$

In this case from $s=\log \frac{\sqrt{F}}{16}$ we should have:

$k_{(s): 1}<\sqrt[3]{10^{2 i}}$

Therefore:

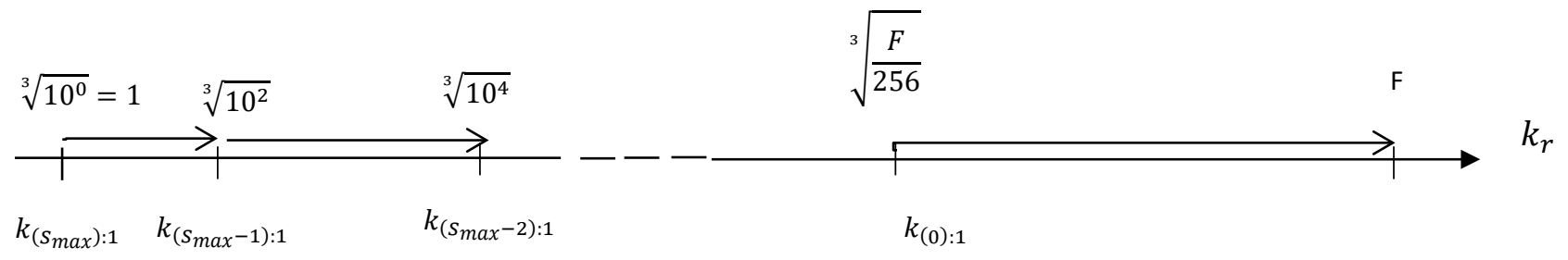

For calculated the value of $P_{(s): 1}$, we should have:

$\left\{\begin{array}{rl}P_{(s): 1} & =\sqrt{\frac{F}{k_{(s): 1}}} \\ k_{(s): 1} & =\sqrt[3]{\left(\frac{1}{4}\right)^{4} \frac{F}{10^{2 s}}}\end{array} \quad=>P_{(s): 1}=\sqrt[3]{16 \times 10^{s} \times F}\right.$

$s_{\max }=\left\lceil\log \frac{\sqrt{F}}{16}\right\rceil, s=s_{\max }-i \quad=>\quad P_{(s): 1}>\frac{\sqrt{F}}{\sqrt[3]{10^{i}}}$

Therefore: 
1

$\frac{\sqrt{F}}{\sqrt[3]{10^{2}}} \quad \frac{\sqrt{F}}{\sqrt[3]{10}} \quad \frac{\sqrt{F}}{\sqrt[3]{10^{0}}}=\sqrt{F}$
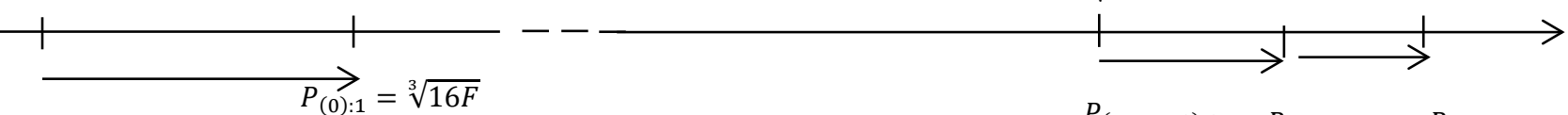

$(P)$

Since $s_{\max }=\left\lceil\log \frac{\sqrt{F}}{16}\right]$, thus for any $s_{\max }$, we have $256 \times 10^{2\left(s_{\max }-1\right)}<F \leq 256 \times 10^{2 s_{\max }}$

Therefore in $\beta(k, 1)$ test we can do as bellow:

$k_{(s): 1}<k_{r}<k_{(s-1): 1}=>n=10^{s}=>k=10^{s} \mathrm{~m}$

If $k_{r}=n^{2}$, by choose $1 \leq n<\sqrt[3]{10}$, then $k_{r}$ located in $s_{\max }$ zone because we have $<\sqrt[3]{100}$.

We can observe in any s zone for a natural number as $F$, when the value of $F$ increases, then the difference between $\sqrt{F}$ and $p_{(s): 1}$ increases, because :

$\sqrt{F}-p_{(s): 1}=\sqrt{F}-p_{\left(s_{\max }-i\right): 1}=\sqrt{F}-\frac{F}{\sqrt[3]{10^{i}}}=\sqrt{F}\left(1-\frac{1}{\sqrt[3]{10^{i}}}\right)$

\section{Introducing $\alpha-s$ Sieve}

Since for each odd composite number we have:

$\left\{\begin{array}{l}F=p q \quad, \quad 3 \leq p \leq q \\ P=2 x+1, \quad q=2 y+1\end{array} \Rightarrow F=4 x y+2(x+y)+1=2 k+1\right.$

Therefore, if we propose $S=x+y+1$ and $R=x y$, then we will have:

$K=\frac{F-1}{2}=2 x y+x+y=2 R+S-1$

By assuming $\alpha=S^{2}-F$ so that $\alpha$ becomes a square number, we will to have:

$S=\frac{p+q}{2} \Rightarrow \alpha=S^{2}-F=\left(\frac{p+q}{2}\right)^{2}-p q=\left(\frac{p-q}{2}\right)^{2}$

In general case, for each value of S, we can obtain its corresponding $p$ as follows:

$S=\frac{p+q}{2} \Rightarrow q=2 S-p \Rightarrow F=p q=p(2 S-p) \Rightarrow\left\{\begin{array}{l}P=S-\sqrt{S^{2}-F} \\ q=S+\sqrt{S^{2}-F}\end{array}\right.$

Therefore by assuming $\alpha=S^{2}-F=t^{2}$, we will have $P=S-t$ and $q=S+t$.

For any $F=p$ q we can conclude that $S=\frac{F+p^{2}}{2 p}$ or $S=\left(\frac{k_{r}+1}{2}\right) \sqrt{\frac{F}{k_{r}}}$ and for any of the two different values of $P$, we have: 
$p_{1}<p_{2} \Rightarrow S_{p_{1}}>S_{p_{2}} \Rightarrow \Delta S_{p_{1}, p_{2}}=S_{p_{1}}-S_{p_{2}}=\frac{\Delta p}{2}\left(\frac{F}{p_{1} p_{2}}-1\right)$

It is seen that for one definite number as $F$ and a constant value of $\Delta p$, with increase in values of $p_{1}$ and $p_{2}$, the $\Delta S$ is decreased. In other words, with decrease in $p$ values, the $S_{p}$ values, increase.

When the difference between $S_{p}$ and $S_{\sqrt{F}}$ is represented by $n$, we will have:

$n=S_{p}-S_{\sqrt{F}}=\frac{F+p^{2}}{2 p}-\sqrt{F} \Rightarrow n=\frac{(\sqrt{F}-p)^{2}}{2 p} \Rightarrow\left\{\begin{array}{l}p=(n+\sqrt{F})-\sqrt{(n+\sqrt{F})^{2}-F} \\ q=(n+\sqrt{F})+\sqrt{(n+\sqrt{F})^{2}-F}\end{array}\right.$

In this case we have:

$S=\frac{p+q}{2}=\lfloor\sqrt{F}\rfloor+n \Rightarrow \alpha=S^{2}-F=t^{2}=\left(\frac{p-q}{2}\right)^{2}$

By considering the points mentioned, we can recommend a sieve to identify an odd composite number is prime or composite. This is based on the premise that if we are able to find values of $S$ in a way that the value of $\alpha$ becomes square, therefore $F$ will be a composite number.

By considering the relation $K=2 R+S-1$ we will have:

$\mid \begin{aligned} & k=\text { an odd number } \Rightarrow S=\text { an even number } \\ & k=\text { an even number } \Rightarrow S=\text { an odd number }\end{aligned}$

The process of calculating the value of $\alpha=S^{2}-F$ and establishing the fact that whether it is square numbers or not, is called $\alpha$ test. Any interval of 10 consecutive $S$ is called the test domain. The reason for defining such a concept as the test domain is due to the repetition of the first digit on the right side of $S$ values in it. For any natural odd number as $F$, considering the oddness or evenness of $K$ and the digit on the right side of it, a unique array for values of $S$ can be stated as follows; in which o represents the oddness of $S$ and e represents the evenness of it. Therefore for any odd number in the form of $F=\bar{\ldots} f_{2} f_{1}$ on the condition that $K$ is even or odd, we define $S_{e \text { or o }}^{f_{1}}$ as

$S_{e \text { or o }}^{f_{1}}=$ (digits on the right side of $S$ in one domain).

Therefore, for different cases, we have:

$$
\begin{gathered}
F=2 k+1=\overline{\ldots 1} \Rightarrow\left\{\begin{array}{l}
k=\text { odd }, S_{e}^{1}=(0,4,6) \\
k=\text { even }, S_{o}^{1}=(1,5,9)
\end{array}\right. \\
F=2 k+1=\overline{\ldots 3} \Rightarrow\left\{\begin{array}{l}
k=\text { odd }, \quad S_{e}^{3}=(2,8) \\
k=\text { even }, S_{o}^{3}=(3,7)
\end{array}\right.
\end{gathered}
$$




$$
\begin{aligned}
& F=2 k+1=\overline{. .7} \Rightarrow \begin{cases}k=\text { odd }, & S_{e}^{7}=(4,6) \\
k=\text { even }, & S_{o}^{7}=(1,9)\end{cases} \\
& F=2 k+1=\overline{. .9} \Rightarrow \begin{cases}k=\text { odd } \quad, & S_{e}^{9}=(0,2,8) \\
k=\text { even } & S_{o}^{9}=(3,5,7)\end{cases}
\end{aligned}
$$

It is noteworthy to mention that in this sieve by finding the first response point, the compositeness of the $F$ number will become obvious. From now the $S$ of any response point will be represented by $S_{r}$. In this case we will have $S_{r}=S_{\min }+(n-1) \times 10$.

Here $n$ represents the number of the test domain which contains the response point (or $S_{r}$ ) and $S_{\text {min }}$ also is the value of $S$ in first test domain which has a digit on the right side equal to $S_{r}$.Therefore for each response point we will have $S_{r}=\frac{p+q}{2}$.

For any $\alpha$ test, we will eliminate the values of $S$ in test domains as much as possible. In other words, some values of $S$ for which $\alpha$ does not become square number (or perfect square) will be eliminated. These processes of eliminating the $S$ values and reaching the response points are called $\alpha-S$ sieve. When $F$ has more than two numbers as $p$, then the probability of reaching the first response point will be much. One of the significant points about this sieve is the increase in density of the existence probability of $P$ values by decreasing $S$ value. In order to establish the compositeness of an odd number, we only need to reach the first response point.

One of the benefits of this sieve is the fact that in many cases we do not need $\alpha$ test for all values of $S$ in order to identify whether $\alpha$ is a square number. By using only a few digits on the right side of $S$ and $F$, then we will be able to eliminate many values of $S$. If $\alpha=\overline{e_{m} e_{m-1} \ldots e_{3} e_{2} e_{1}}$ is a prefect square number, then we will be able to use the following notes in this sieve.

Note 3: $e_{1}$ can only have the values as $0,1,4,5,6,9$.

Note 4: If $e_{1}=5$, then $E_{3}=\overline{e_{3} e_{2} e_{1}}$ must be as one of the member of the set $\{025,225,625\}$. For proving, by assume $\alpha=\left(\overline{\ldots l_{3} l_{2} l_{1}}\right)^{2}$ we should have:

$$
\begin{aligned}
& \left(\overline{l_{3} l_{2} l_{1}}\right)^{2}=\left(\overline{l_{3} l_{2} 5}\right)^{2}=10^{4} l_{3}^{2}+\left(2 l_{1} l_{3}+l_{3}\right) \times 10^{3}+\left(l_{2}^{2}+l_{2}\right) \times 10^{2}+5^{2} \\
& \Rightarrow\left\{\begin{array}{l}
\overline{e_{2} e_{1}}=5^{2}=\overline{25} \\
e_{3}=l_{2}^{2}+l_{2}, l_{2}: 0 \rightarrow 9 \Rightarrow e_{3} \in\{0,2,6\}
\end{array}\right.
\end{aligned}
$$

Note 5: If $e_{1}=0$, then $E_{3}=\overline{e_{3} e_{2} e_{1}}$ will necessarily a member of the set $\{000,100,400,500,600,900\}$.

For perfect square numbers with the digit zero on their right side, the number of zeros on their right side must always be even.

Note 6: In the case that $\alpha$ is an even number, we have $E_{3}-0=8 m$ or $E_{3}-4=8 m$ and in the case 
that $\alpha$ is an odd number, we have $E_{3}-1=8 \mathrm{~m}$.

Therefore, by selecting $E_{N}$ for big value of $N$ we can eliminate more test points in a way that fewer test points will remain for $\alpha$ test and this is very suitable for large numbers. In doing so, we will utilize the following theorems.

Theorem 7: If the natural number as $\alpha=\overline{e_{M} e_{M-1} \ldots e_{3} e_{2} e_{1}}$ is a square number and the $N$ digit on the right side is represented by $E_{N}=\overline{e_{N} e_{N-1} \ldots e_{2} e_{1}}$, then in a way that $\alpha$ is odd, we have:

$E_{N}-(2 i-1)^{2}=2^{N} m_{i} \quad\left(1 \leq i \leq 2^{N-3}, N>3\right)$

and in a way that $\alpha$ is even, we have:

$E_{N}-(2 i-2)^{2}=2^{N} m_{i} \quad\left(1 \leq i \leq 2^{N-3}, N>3\right)$

( $m_{i}$ is a natural number)

Proof: Since any natural number can be represented by $2 k_{r}+l_{r}$ in a way that if the number is even, then $l_{r}=0$ and if it is odd, then $l_{r}=1$ and that $k_{r}$ is a non-negative integer number; therefore, by assuming that $\Delta$ is a perfect square number, we will have:

$\alpha=\overline{e_{M} e_{M-1} \ldots e_{3} e_{2} e_{1}}=\left(2 k_{1}+l_{1}\right)^{2}=\left(2\left(2 k_{2}+l_{2}\right)+l_{1}\right)^{2}=\left(2\left(2\left(2 k_{3}+l_{3}\right)+l_{2}\right)+l_{1}\right)^{2}$

$=\cdots=\left[2^{N} k_{N}+\left(2^{N-1} \times l_{N}+2^{N-2} \times l_{N-1}+\cdots+2 l_{2}+l_{1}\right)\right]^{2}$

We will continue the operation above until the value of $k_{r}$ in $2 k_{r}+l_{r}$ equal to 1.

When we assume:

$A=2^{N-1} \times l_{N}+2^{N-2} \times l_{N-1}+000+2 l_{2}+l_{1}$

It can be seen that A equivalent to a number in base 2 as below:

$A=\left(\overline{l_{N} l_{N-1} \ldots l_{3} l_{2} l_{1}}\right)_{2}$

Therefore:

$\alpha=\left(2^{N} k_{N}+A\right)^{2}=\left(2^{N} k_{N}^{2}+2 k_{N} A\right) \times 2^{N}+A^{2}$

By assuming $T=2^{N} k_{N}^{2}+2 k_{N} A$,we will have:

$$
\alpha=T \times 2^{N}+A^{2}
$$

On the other hand, we can write:

$\alpha=\overline{e_{M} e_{M-1} \ldots e_{3} e_{2} e_{1}}=\overline{e_{M} e_{M-1} \cdots e_{N+1}} \times 10^{N}+\overline{e_{N} e_{N-1} \ldots e_{1}}$

By assuming $E_{N}=\overline{e_{N} e_{N-1} \ldots e_{1}}$ and $S=\overline{e_{M} e_{M-1} \ldots e_{N+1}}$, we will have:

$\alpha=S \times 10^{N}+E_{N}$ 
From (1) and (2) we can conclude that:

$E_{N}-A^{2}=\left(T-S \times 5^{N}\right) \times 2^{N}=2^{N} \times Q$

It means that $2^{N}$ counts the number $E_{N}-A^{2}$.

Since we have:

$A_{\max }=(\overline{111 \ldots 1})_{2}=2^{N-1}+2^{N-2}+\cdots+2+1 \Rightarrow A_{\max }=2^{N}-1$

Therefore the number of the tests that we can do in a definite $E_{N}$ will be as below:

$\mid \begin{aligned} & E_{N}-1^{2}=2^{N} m_{1} \\ & E_{N}-3^{2}=2^{N} m_{2} \\ & \cdots \cdots \ldots \ldots \ldots \ldots \ldots \ldots \\ & E_{N}-\left(2 n_{1}-1\right)^{2}=2^{N} m_{n_{1}}\end{aligned}$

Thus we will have:

$2^{N}-1=2 n_{1}-1 \Rightarrow n_{1}=2^{N-1}$

Therefore:

$E_{N}-(2 i-1)^{2}=2^{N} m_{i} \quad, \quad 1 \leq i \leq 2^{N-1}$

For the case that $\alpha$ is even, we have $l_{1}=0$ and its proof is like the previous one; then for this case we will have:

$A_{\max }=(\overline{111 \ldots \overline{1}})_{2}=2^{N-1}+2^{N-2}+\cdots+2+0 \Rightarrow A_{\max }=2^{N}-2$

Therefore, the number of $E_{N}$ tests, when $\Delta$ is even, will be as below:

$\mid \begin{aligned} & E_{N}-0^{2}=2^{N} m_{1} \\ & E_{N}-2^{2}=2^{N} m_{2} \\ & \cdots \cdots \cdots \cdots \cdots \cdots \cdots \\ & E_{N}-\left(2 n_{1}-2\right)^{2}=2^{N} m_{n_{1}}\end{aligned}$

Thus we have:

$2^{N}-2=2 n_{1}-2 \Rightarrow n_{1}=2^{N-1}$

Then in general case we will have:

$$
E_{N}-(2 i-2)^{2}=2^{N} m \quad, 1 \leq i \leq 2^{N-1}
$$

To continue the proof, we first consider a case in which $\alpha$ is odd and for a definite $E_{N}$ test with the arrangement of A values in ascending order, therefore we should have: 


$$
\mid \begin{aligned}
& E_{N}-1^{2}=2^{N} m_{1} \\
& E_{N}-3^{2}=2^{N} m_{2} \\
& \ldots \ldots \ldots \ldots \ldots \ldots \ldots \\
& E_{N}-(2 x-1)^{2}=2^{N} m_{x} \\
& \ldots \ldots \ldots \ldots \ldots \ldots \ldots \\
& E_{N}-(2 y-1)^{2}=2^{N} m_{y} \\
& \cdots \cdots \ldots \ldots \ldots \ldots \ldots \ldots \\
& E_{N}-\left(2 n_{1}-1\right)^{2}=2^{N} m_{n_{1}}
\end{aligned}
$$

Since we have $\Delta A_{\max }=\left(2 n_{1}-1\right)-(-1)=2 n_{1}=2^{N}$ therefore for two value of $A$ with difference $2^{N-1}$ so that $(2 x-1)=2 \times 2^{N-2}-i$ and $(2 y-1)=2 \times 2^{N-1}-i$ (when $i$ is a odd number and located in interval from 1 to $2^{N-1}-1$ ) then we have:

$(2 y-1)^{2}-(2 x-1)^{2}=\left(2^{N-1}\right) \times(2 x+2 y-2)=2^{N}(y+x-1)$

Therefore the number of $E_{N}$ tests can be calculated by $n_{2}=2^{N-2}$.

In an ascending arrangement of the A values, for both of its values which lie in a symmetrical position, we will have:

$$
\begin{aligned}
& \mid \begin{array}{l}
E_{N}-1^{2}=2^{N} m_{1} \\
E_{N}-3^{2}=2^{N} m_{2} \\
\ldots \ldots \ldots \ldots \ldots \ldots \ldots \\
E_{N}-[2(i+1)-1]^{2}=2^{N} m_{i+1} \\
\ldots \ldots \ldots \ldots \ldots \ldots . . \\
E_{N}-\left[2\left(n_{2}-i\right)-1\right]^{2}=2^{N} m_{n_{2}-i} \\
\ldots \ldots \ldots \ldots \ldots \ldots \ldots \\
E_{N}-\left[2 n_{2}-3\right]^{2}=2^{N} m_{n_{2}-1} \\
E_{N}-\left[2 n_{2}-1\right]^{2}=2^{N} m_{n_{2}}
\end{array} \\
& \Rightarrow \quad\left[2\left(n_{2}-i\right)-1\right]^{2}-[2(i+1)-1]^{2}=\left(2 n_{2}-4 i-2\right)\left(2 n_{2}\right) \\
& =\left(2 \times 2^{N-2}-4 i-2\right)\left(2 \times 2^{N-2}\right)=\left(2^{N-2}-2 i-1\right) \times 2^{N}
\end{aligned}
$$

Since the result of $\left(2^{N-2}-2 i-1\right)$ is positive, then $N$ must be a number greater than 3 . Therefore the total number of the remaining values of A decreases from $2^{N-2}$ to $2^{N-3}$, which is represented by $n_{N}$. Thus in general case when $\alpha$ is odd, for every $E_{N}$ test we will have:

$\mid \begin{aligned} & E_{N}-1^{2}=2^{N} m_{1} \\ & E_{N}-3^{2}=2^{N} m_{2} \\ & \cdots \cdots \cdots \cdots \cdots \cdots \cdots \\ & E_{N}-\left(2 n_{N}-1\right)^{2}=2^{N} m_{n_{N}}\end{aligned}$

Then the total number of $E_{N}$ tests equal to $n_{N}=2^{N-3}$.

For the case in which $\alpha$ is even, the proof process is completely similar and for each $E_{N}$ test we have: 
$\mid \begin{aligned} & E_{N}-0^{2}=2^{N} m_{1} \\ & E_{N}-2^{2}=2^{N} m_{2} \\ & \ldots \ldots \ldots \ldots \ldots \ldots \ldots \\ & E_{N}-\left(2 n_{N}-2\right)^{2}=2^{N} m_{n_{N}}\end{aligned}$

In a way that the total number of each $E_{N}$ test is calculated like that of the previous case, thus we will have $n_{N}=2^{N-3}$, therefore the proof is complete.

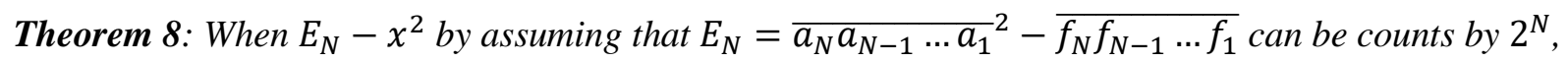
and when we replace $a_{N}$ with another digit like as $a_{N}^{\prime}$ and represent the result of ${\overline{a_{N}^{\prime} a_{N-1} \ldots a_{1}}}^{2}-$ $\overline{f_{N} f_{N-1} \cdots f_{1}}$ by $E_{N}^{\prime}$, therefore $E_{N}^{\prime}-x^{2}$ can also count $2^{N}$.

Proof: If in $E_{N}=\overline{a_{N} a_{N-1} \ldots a_{1}^{2}}-\overline{f_{N} f_{N-1} \ldots f_{1}}=2^{N}+x^{2}$, we replace $a_{N}$ with the digit $a_{N}^{\prime}$ in a way that a $\dot{a}_{N}=a_{N} \pm r$, and $r$ can have one of the digits from 1 to 9 , then we will have:

$$
\begin{aligned}
& E_{N}^{\prime}={\overline{a_{N}^{\prime} a_{N-1} \ldots a_{1}}}^{2}-\overline{f_{N} f_{N-1} \ldots f_{1}}=\left(\overline{\left.a_{N} \pm r\right) a_{N-1} \ldots a_{1}}-\overline{f_{N} f_{N-1} \ldots f_{1}}\right. \\
& =\left(\overline{\left.a_{N} \pm r\right) a_{N-1} \ldots a_{1}}-\overline{f_{N} f_{N-1} \ldots f_{1}}=\left(( \pm r) \times 10^{N-1}+\overline{a_{N} a_{N-1} \ldots a_{1}}\right)^{2}-\overline{f_{N} f_{N-1} \ldots f_{1}}\right. \\
& =r^{2} \times 10^{2 N-2} \pm 2 r \times 10^{N-1} \times \overline{a_{N} a_{N-1} \ldots a_{1}}+\overline{a_{N} a_{N-1} \ldots \bar{a}_{1}}-\overline{f_{N} f_{N-1} \ldots f_{1}} \\
& .=\left(r^{2} \times 5^{2 N-2} \times 2^{N-2} \pm r \times 5^{N-2} \times \overline{a_{N} a_{N-1} \ldots a_{1}}\right) \times 2^{N}+{\overline{a_{N} a_{N-1} \ldots a_{1}}}^{2}-\overline{f_{N} f_{N-1} \ldots f_{1}}
\end{aligned}
$$

By assuming $T=r^{2} \times 5^{2 N-2} \times 2^{N-2} \pm r \times 5^{N-2} \times \overline{a_{N} a_{N-1} \ldots a_{1}}$, we will have:

$E_{N}^{\prime}=T \times 2^{N}+E_{N} \Rightarrow E_{N}^{\prime}=T \times 2^{N}+2^{N} m+x^{2} \Rightarrow E_{N}^{\prime}-x^{2}=(T+m) \times 2^{N}$

It means $E_{N}^{\prime}-x^{2}$ can be count by $2^{N}$ and the proof is complete.

Note 7: When the values of $E_{N}$ are even, we can decrease the number of $E_{N}$ test. As an example, for $E_{5}$ to $E_{7}$ we have:

$$
N=5 \Rightarrow \begin{aligned}
& E_{5}-0^{2}=32 m \\
& E_{5}-2^{2}=32 m \\
& E_{5}-4^{2}=32 m
\end{aligned}, N=6 \Rightarrow \begin{aligned}
& E_{6}-0^{2}=64 m \\
& E_{6}-2^{2}=64 m \\
& E_{6}-4^{2}=64 m \\
& E_{6}-6^{2}=64 m
\end{aligned}, N=7 \Rightarrow \begin{aligned}
& E_{7}-0^{2}=128 m \\
& E_{7}-2^{2}=128 m \\
& E_{7}-4^{2}=128 m \\
& E_{7}-6^{2}=128 m \\
& E_{7}-8^{2}=128 m \\
& E_{7}-10^{2}=128 m \\
& E_{7}-14^{2}=128 m
\end{aligned}
$$

In this sieve we can use theorems 7 and 8 in $E_{N}$ tests as below.

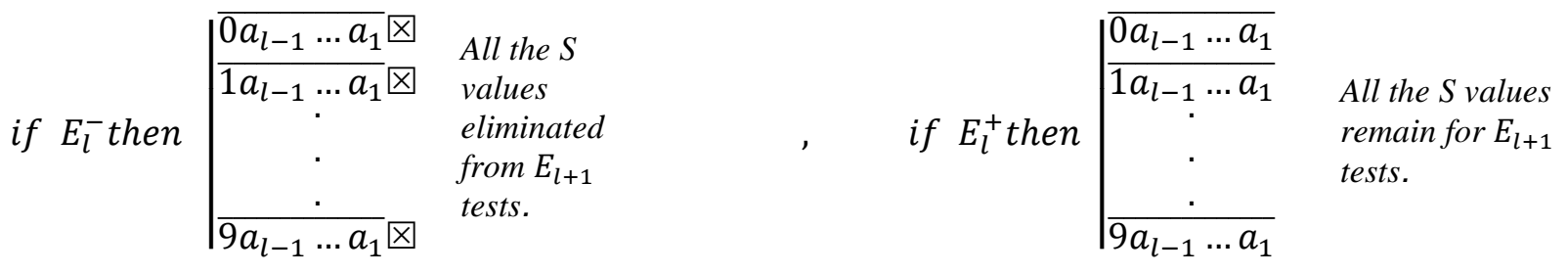


Theorem 9: When $E_{N}$ test for a definite $S$ is positive, then $E_{N-r}$ test is positive for that $S$.

Proof: From theorem (4) we have:

$E_{N}-x^{2}=2^{N} m \Rightarrow \overline{e_{N} e_{N-1} \ldots e_{1}}-x^{2}=2^{N} m_{1}$

$\Rightarrow \overline{e_{N} e_{N-1} \ldots e_{N-r+1}} \times 10^{N-r}+\overline{e_{N-l} \ldots e_{1}}-x^{2}=2^{N} m_{1}$

$\Rightarrow \overline{e_{N-l} \ldots e_{1}}-x^{2}=2^{N} m_{1}-\overline{e_{N} e_{N-1} \ldots e_{N-r+1}} \times 5^{N-r} \times 2^{N-r}$

$.=2^{N-i}\left(2^{i} m_{1}-\overline{e_{N} e_{N-1} \cdots e_{N-r+1}} \times 5^{N-r}\right)=2^{N-r} m_{2} \Rightarrow E_{N-r}-x^{2}=2^{N-r} m_{2}$

It means the number $2^{N-r}$ counts $E_{N-r}-x^{2}$. In other words $E_{N-r}$ test is positive and the proof is complete.

Therefore in every $E_{N}$ test for a definite $S$ except for values of $x$ for which $E_{N-1}$ test is positive, all the tests related to the other values of $x$ are eliminated from the $E_{N}$ tests. Thus total number of $E_{N}$ tests can be calculated as follows:

$n_{E_{N}} \approx 2^{N-3}-2^{N-4}+1=2^{N-4}+1$

Theorem 10: If $E_{N}-x^{2}=2^{N} m$, i.e. $E_{N}$ test will be positive, then $E_{M}-x^{2}$ for each $M$ greater than $N$ can be count by $2^{N}$.

\section{Proof:}

$$
\begin{aligned}
& E_{N}-x^{2}=2^{N} m \Rightarrow \overline{e_{N} e_{N-1} \cdots e_{1}}-x^{2}=2^{N} m_{1} \Rightarrow \overline{e_{M} e_{M-1} \cdots e_{N+1}} \times 10^{N}+\overline{e_{N} e_{N-1} \cdots e_{1}}-x^{2} \\
& =2^{N} m_{1}+\overline{e_{M} e_{M-1} \cdots e_{N+1}} \times 10^{N}=2^{N}\left(m_{1}+\overline{e_{M} e_{M-1} \cdots e_{N+1}} \times 5^{N}\right)=2^{N} m_{2} \\
& \Rightarrow \overline{e_{M} e_{M-1} \cdots e_{2} e_{1}}-x^{2}=2^{N} m_{2} \Rightarrow E_{M}-x^{2}=2^{N} m_{2}
\end{aligned}
$$

It means that the $2^{N}$ counts $E_{M}-x^{2}$ and the proof is complete.

In $E_{N}$ tests for a definite $S$, only for a one value of $x$, the test result is positive. It is because if we assume for the two different values $x_{1}$ and $x_{2}$ the test result is positive, then it means $x_{1}$ and $x_{2}$ will have the same outcome.

Theorem 11: If values of $E_{N}$ for two values of $S$ with a difference equal to10,from the two domains of the consecutive test is represented by $E_{N(i+1)}$ and $E_{N(i)}$ in a way that $i$ represents the number of the test domain, then values of $\Delta E_{N(i, i+1)}=E_{N(i+1)}-E_{N(i)}$ for the two consecutive domains of the $E_{N}$ tests form an arithmetic progression with 200 as common difference.

Proof: From $E_{N}$ definition, we have:

$$
\left\{\begin{array}{l}
E_{N(1)}=S_{\text {min }}^{2}-\overline{f_{N} \ldots f_{1}} \\
E_{N(i)}=\left(S_{\min }+10(i-1)\right)^{2}-\overline{f_{N} \ldots f_{1}}
\end{array} \Rightarrow \quad E_{N(i)}=E_{N(1)}+20(i-1) S_{\min }+100(i-1)^{2}\right.
$$




$$
\begin{aligned}
& \Rightarrow\left\{\begin{array} { l } 
{ E _ { N ( i + 1 ) } = E _ { N ( 1 ) } + 2 0 i S _ { m i n } + 1 0 0 i ^ { 2 } } \\
{ E _ { N ( i ) } = E _ { ( N ) 1 } + 2 0 ( i - 1 ) S _ { \operatorname { m i n } } + 1 0 0 ( i - 1 ) ^ { 2 } } \\
{ E _ { N ( i - 1 ) } = E _ { N ( 1 ) } + 2 0 ( i - 2 ) S _ { \operatorname { m i n } } + 1 0 0 ( i - 2 ) ^ { 2 } }
\end{array} \Rightarrow \left\{\begin{array}{l}
\Delta E_{N(i-1, i)}=20 S_{\min }+100(2 i-3) \\
\Delta E_{N(i, i+1)}=20 S_{\min }+100(2 i-1)
\end{array}\right.\right. \\
& \Rightarrow \Delta E_{N(i, i+1)}-\Delta E_{N(i-1, i)}=200
\end{aligned}
$$

It means the values of $\Delta E_{N(i, i+1)}$ forms an arithmetic progression with 200 as common difference value.

Therefore we will have:

$X=\Delta E_{N(1,2)} \stackrel{+200}{\longrightarrow} \Delta E_{N(2,3)} \stackrel{+200}{\longrightarrow} \Delta E_{N(3,4)} \stackrel{+200}{\longrightarrow} \ldots$

$\Rightarrow E_{N(1)} \stackrel{X}{\rightarrow} E_{N(2)} \stackrel{X+200}{\longrightarrow} E_{N(3)} \stackrel{X+2 \times 200}{\longrightarrow} E_{N(4)} \stackrel{X+3 \times 200}{\longrightarrow} E_{N(5)} \rightarrow \cdots$

$\Rightarrow E_{N(i)}-E_{N(i-1)}=X+(i-2) \times 200 \Rightarrow E_{N(i)}=E_{N(1)}+(i-1) X+(i-1)(i-2) \times 100$

If we represent the $i$ th group of the $S$ values with the number $10^{N}$ by $H_{N(i)}$ and the $j$ th group of the $S$ values with the number $10^{N-1}$ by $A_{N(j)}$, in a way that $i$ in them is from 1 to $\left\lceil\frac{S_{\max }-S_{\min }}{10^{N}}\right\rceil$ and $j$ is from 1 to 10 , then according to theorem 8 it will suffice to do the $E_{N}$ tests only for $A_{N(1)}$, i.e. the first group with the number $10^{N-1}$ in the $H_{N(1)}$ group. If the test result for one definite $S$ in $A_{N(1)}$ will be positive, it means the result of this test is positive for all the $S$ values that contain $N$-1 similar digits on the right side and are only different in the first digit on the left side and are located in other nine groups of A. But if the result of $E_{N}$ test is negative for one of the $S$ values in $A_{N(1)}$, then this test is negative for all the $S$ values which are only different in their first digit on the left side and are located in the other nine groups of A. If in the first $A_{N(1)}$ all the tests of $E_{N}$ are negative, it means that the odd number under the test is a prime number.

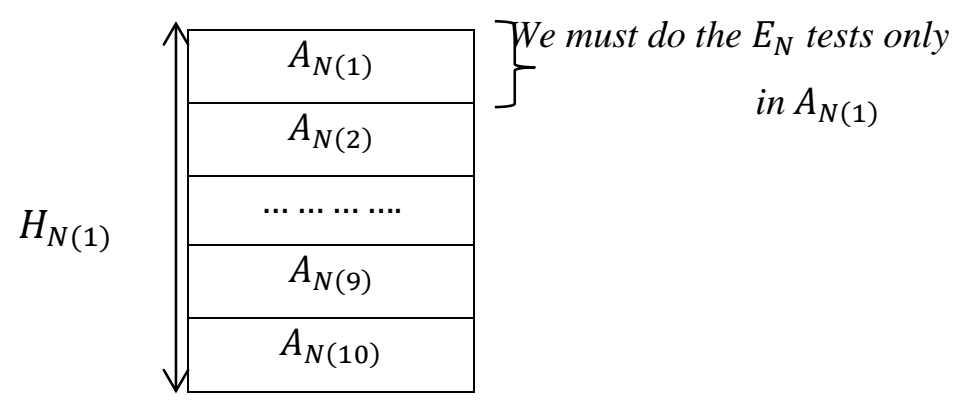

If we assume that the number of $E_{N}^{+}$tests in $A_{N(1)}$ is equal to w, then the total number of $\alpha$ tests in $\alpha-S$ sieve can be calculated by $n_{\alpha-E_{N}^{+}}=\left\lceil\frac{S_{\max }-S_{\min }}{10^{N}}\right\rceil \times 10 \mathrm{w}$.

Note 8: If $\alpha=\overline{e_{m} e_{m-1} \ldots e_{3} e_{2} e_{1}}$ will be a prefect square number, then we must to have: $\sum_{i=1}^{m} e_{i}=9 k^{2} \sum_{i=1}^{m} e_{i}=3 k+1$ or 
In the $\alpha-S$ sieve it is better to do the $E_{N}$ tests for the two value of $N_{1}$ and $N_{2}$ in which $N_{1}$ is less than $N_{2}$ so that we should have fewer $E_{N}$ tests.

\begin{tabular}{|c|c|c|c|c|}
\hline$D$ & $S$ & $E_{N_{1}}$ & $E_{N_{2}}$ & test $\alpha$ \\
\hline$D_{1}$ & & & & \\
\hline$D_{2}$ & & & & \\
\hline 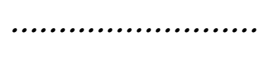 & 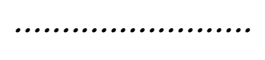 & 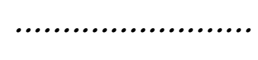 & 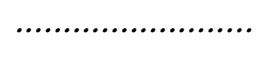 & 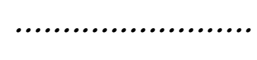 \\
\hline
\end{tabular}

If for very large numbers, $E_{N}$ tests are done by bigger value of $N$,Then the number of $\alpha$ tests will be decreased. It should be noted that working on some digits on the right side of a large number is much easier than working on all of its digits and takes much less time. We can observe the number of $E_{N}$ tests will be rigorous decreased, which is one of the important properties of this sieve.

Example 2: Show that $F=251,953,878,652,772,860,514,325,499,229$ is a composite number.

Answer

$\lfloor\sqrt{F}\rfloor=501,950,075,865,935, k=\frac{F-1}{2}=$ even $, S_{\text {min }}=501,950,075,865,927 \Rightarrow S_{O}^{9}=(3,5,7)$

\begin{tabular}{|c|l|l|l|}
\hline \multicolumn{2}{|c|}{ The first set of $S$ by $10^{3}$ length } & \multicolumn{1}{|c|}{$E_{3}$} & $E_{5}$ \\
$E_{5}$
\end{tabular}




\begin{tabular}{|c|c|c|c|}
\hline$D_{5}$ & $\begin{array}{l}501,950,075,865,967 \\
501,950,075,865,973 \\
501,950,075,865,975\end{array}$ & $\begin{array}{l}E_{3}=\overline{860} \rightarrow \bowtie \\
E_{3}=\overline{500} \rightarrow \bigotimes \\
E_{3}=\overline{396} \rightarrow E_{3}^{+}\end{array}$ & $E_{5}^{-} \rightarrow \bowtie$ \\
\hline
\end{tabular}

It can be seen that for $S=504,037,195,361,823, \alpha$ is a perfect square number and for this first $S_{r}$ we have:

$S_{r}=504,037,195,361,823 \rightarrow \alpha=S_{r}^{2}-F=t^{2} \Rightarrow \Rightarrow\left\{\begin{array}{l}p=458,215,632,147,113 \\ q=s 594,858,758,576,533\end{array}\right.$

It is observed that only by $E_{5}$ test , the $S$ values are eliminated from 5 domains.

The most important notes regarding to the $\alpha-S$ sieve are mentioned as follows:

1. There is no need to know the prime numbers less than the square root of the number under test.

2. The results of $E_{N}$ tests do not depend on the largeness of the numbers under test. They only depend on the type and the arrangement of $N$ digits on the right side.

3. When we use $E_{N}$ test for $N$ digits on the right side of a definite $S$ in a way that the test result becomes negative, all the $S$ values which contain $N-1$ similar digits on the right side, will be eliminated from the $\alpha$ test.

4. For large numbers, by this sieve, we will reach the answer more quickly and more easily than by tests divisibility test for prime numbers less than its square root. The largeness of the number allows us to do $E_{N}$ tests for greater $N$ value.

5. We will not need time-consuming and big computations with this sieve because in $E_{N}$ tests we only use $N$ digits on the right side of the numbers.

\section{Introduce $\beta-s$ Sieve}

Whenever by assuming $(1 \leq p \leq q) F=p q$ and $(1 \leq n \leq m) k=m n$ the result of $\beta(k, \theta)$ is a square number and by representing the phrase $\lfloor\sqrt{4 k F}\rfloor+\theta$ by $S$, therefore the values of $p$ and $q$ can be calculated as follows:

$\beta(k, \theta)=S^{2}-4 k F=t^{2} \Rightarrow \mid \begin{aligned} & p=\frac{S \mp t}{2 m} \\ & q=\frac{S \pm t}{2 n}\end{aligned}$

For any $k$ values we can calculate the values of $S$ and $\beta(k, \theta)$ by placing the consecutive values of natural numbers in $(\theta \geq 1) \theta$. By Considering the theorems and the notes mentioned, from a few number of digits on the right side of $\beta(k, \theta)$ value, we will be able to eliminate many value of $S$ from the test for which the result of $\beta(k, \theta)$ is not square. This method of sieve, in which by eliminating $S$ values, we want that $\beta(k, \theta)$ to be a square number, is called $\beta-S$ sieve. In this sieve, we represent $N$ digits on the right side 
of $\beta(k, \theta)$ value by $E_{N}$ and we do the $E_{N}$ tests like $\alpha-S$ sieve. the sieving $S$ values only from $k_{r}=1$ to $k_{r}=\sqrt[3]{100}$ (in $S_{\max }$ zone), is equal to sieve the $p$ values from $\sqrt{F}$ to $\frac{\sqrt{F}}{\sqrt[3]{100}}$ in probable interval containing $p$ values.This is one of the most important benefits of this sieve. In this sieve for one definite $\theta$ and $K$, we can assume:

$$
\begin{aligned}
& \left\{\begin{array}{l}
\lfloor\sqrt{4 k F}\rfloor+\theta=S=\overline{a_{N} \ldots a_{2} a_{1}} \\
4 k F=\ldots b_{N} \ldots b_{2} b_{1}
\end{array}\right. \\
\Rightarrow & \beta(k, 1)=S^{2}-4 k F=\overline{\ldots e_{N} \ldots e_{2} e_{1}} \Rightarrow E_{N}=\overline{e_{N} \ldots e_{2} e_{1}}
\end{aligned}
$$

If we represent the odd values of $a_{1}$ for $b_{1}$ by $S_{o}^{b_{1}}$ and the even values of $a_{1}$ for $b_{1}$ by $S_{e}^{b_{1}}$, thus we will have:

$$
\begin{gathered}
, b_{1}=2 \Rightarrow\left\{\begin{array}{l}
S_{o}^{2}=(1,9) \\
S_{e}^{2}=(4,6)
\end{array}, \quad b_{1}=4 \Rightarrow\left\{\begin{array}{l}
S_{o}^{4}=(3,5,7) \\
S_{e}^{4}=(0,2,8)
\end{array} b_{1}=0 \Rightarrow\left\{\begin{array}{l}
S_{o}^{0}=(1,3,5,7,9) \\
S_{e}^{0}=(0,2,4,6,8)
\end{array}\right.\right.\right. \\
b_{1}=6 \Rightarrow\left\{\begin{array}{l}
S_{o}^{6}=(1,5,9) \\
S_{e}^{6}=(0,4,6)
\end{array}, \quad b_{1}=8 \Rightarrow\left\{\begin{array}{l}
S_{o}^{8}=(3,7) \\
S_{e}^{8}=(2,8)
\end{array}\right.\right.
\end{gathered}
$$

When the result of $\beta(k, 1)$ is a square number, we will have:

$\beta(k, \theta)=S^{2}-4 k F=(m p+n q)^{2}-4(m n)(p q)=(m p-n q)^{2}=t^{2}$

Therefore, when we have $S=m p+n q$, the value of $\beta(k, \theta)$ will be a square number and the desired $S$ value, will be $S_{r}=m p+n q$. Considering that the values of $m$ and $n$ are odd or even, through $k=m n$ we can determine if the values of $S$ are odd or even.

$$
\begin{gathered}
\left\{\begin{array} { l } 
{ m = \text { odd } } \\
{ n = \text { odd } }
\end{array} \text { or } \left\{\begin{array}{c}
m=\text { even } \\
n=\text { even }
\end{array} \Rightarrow S=\right.\right.\text { even } \\
\left\{\begin{array} { l } 
{ m = \text { odd } } \\
{ n = \text { even } }
\end{array} \text { or } \left\{\begin{array}{c}
m=\text { even } \\
n=\text { odd }
\end{array} \Rightarrow S=\right.\right.\text { odd }
\end{gathered}
$$

In order to use this sieve between two values of $k_{r}$, we will do as follows:

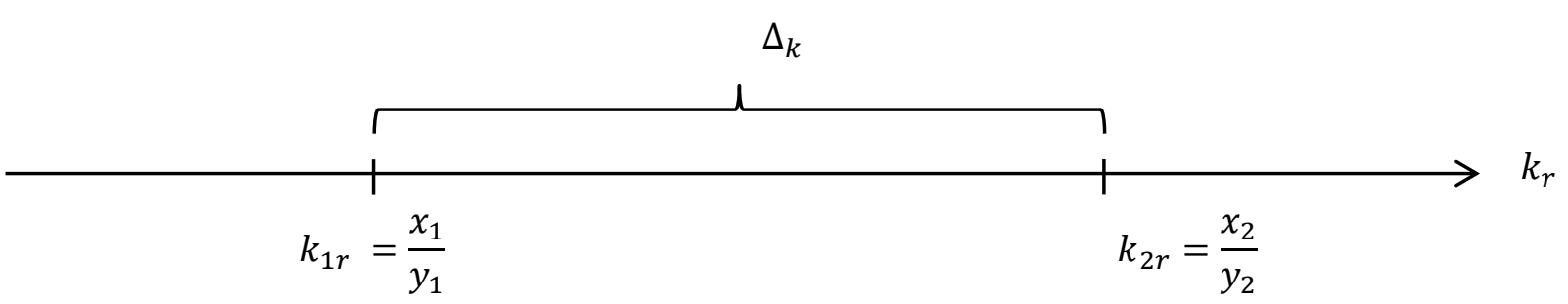

By calculating the minimum value of $S$ in the sieve interval of $\Delta$, we will have:

$$
S_{d}=\lfloor\sqrt{4 k F}\rfloor=\left\lfloor\sqrt{4 x_{1} y_{1} F}\right\rfloor
$$

To calculate the maximum value of $S$ in the sieve interval of $\Delta$, we will do as follows:

$$
k_{r}=\frac{q}{p}=\frac{x_{2}}{y_{2}} \quad, \quad F=k_{r} p^{2} \Rightarrow\left\{\begin{array}{c}
p=\sqrt{\frac{F}{k_{r}}}=\sqrt{\frac{y_{2} F}{x_{2}}} \\
q=\frac{x_{2}}{y} \sqrt{\frac{y_{2} F}{x_{2}}}
\end{array} \Rightarrow S_{u}=x_{1} p+y_{1} q=\left(x_{1}+x_{2}\right) \sqrt{\frac{y_{2} F}{x_{2}}}\right.
$$

In the best case, If we consider $x_{1}$ and $x_{2}$ as consecutive integer number and $y_{1}=y_{2}=y$, then we 
will have:

,$S_{u}=\left(2 x_{1}+1\right) \sqrt{\frac{y F}{x_{1}+1}} S_{d}=\left\lfloor\sqrt{4 x_{1} y F}\right\rfloor$

If one $S_{r}$ is located in the sieve interval of $\Delta$, we represented it by $\Delta_{r}$. For each $S_{r}$ in a sieve interval of $\Delta_{r}$, we have:

$\left\{\begin{array}{l}S_{r}=S_{d}+\theta=m p+n q \\ \theta=\left\lfloor\left(\sqrt{x_{1} p}-\sqrt{y q}\right)^{2}\right\rfloor+1\end{array} \Rightarrow \beta(k, \theta)=(m p-n q)^{2}=t^{2}\right.$

In orders that the sieve should be easier, between the two consecutive integer values of $k_{r}$, especially for big values of $F$, it is better to divide the distance into y equal parts in which each part is an independent sieve zone.

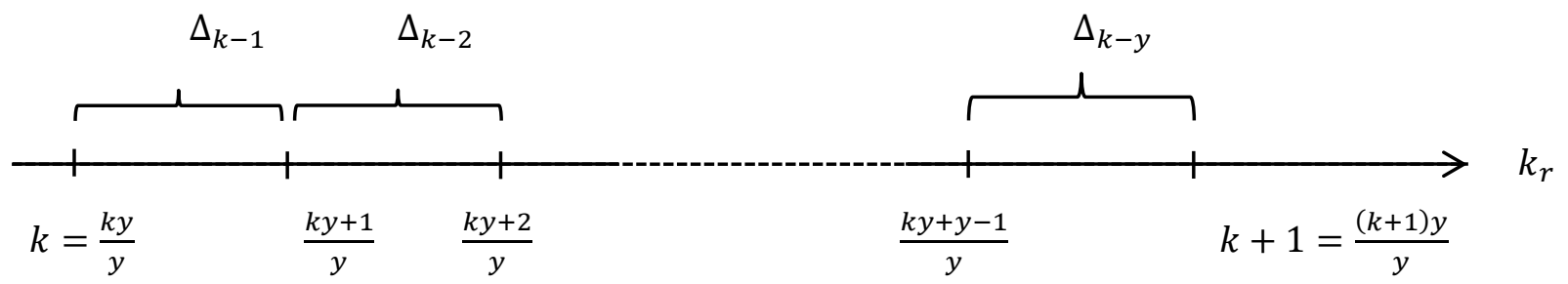

$\Delta_{k-i}$ represents the it $h$ of sieve zone related to $K$. In a particular case when the distance between the two integer values of consecutive $k_{r}$ is selected as one sieve zone, we will do as follows:

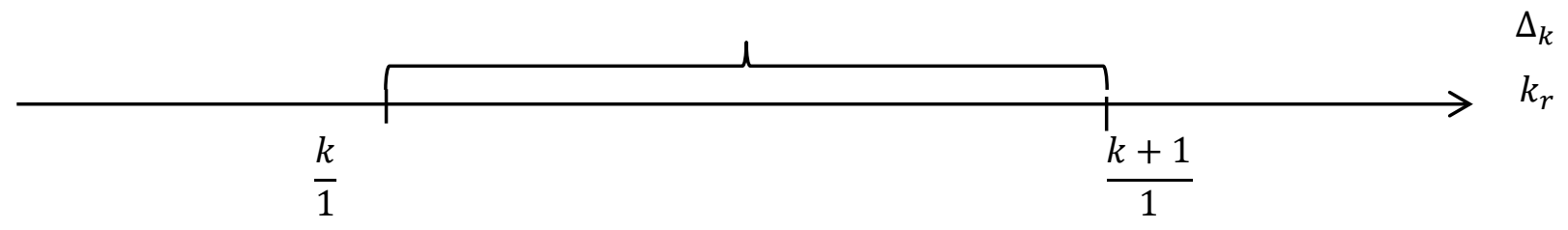

$$
S_{d}=\lfloor\sqrt{4 k F}\rfloor \quad, \quad S_{u}=\left\lceil(2 k+1) \sqrt{\frac{F}{k+1}}\right\rceil
$$

In zones of $\Delta_{k-i}$, the length of the sieve intervals is decreased by increasing the i. In other words:

$L_{\Delta_{k-i}}=S_{u_{k-i}}-S_{d_{k-i}} \Rightarrow L_{\Delta_{k-1}}>L_{\Delta_{k-2}}>L_{\Delta_{k-3}}>\cdots$

Therefore, for the sieve zones between the consecutive integer values of $k_{r}$, the length of the intervals are decreased.

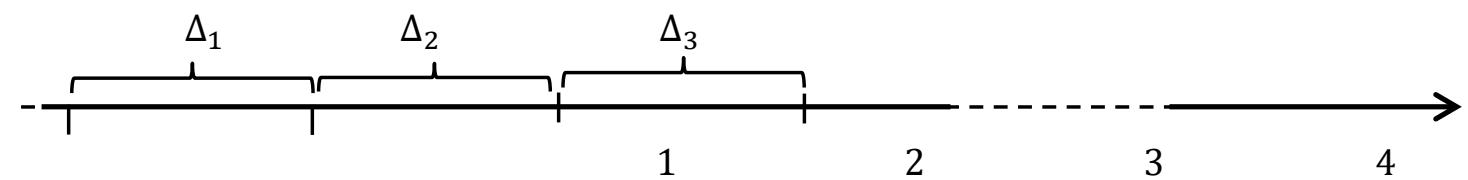

Therefore:

$L_{\Delta_{1}}>L_{\Delta_{2}}>L_{\Delta_{3}}>\cdots$

The decreasing of the length of consecutive sieve zones for $k_{r}$ values which are located in $S=0$ zone ,finally to get at zero. In a way that for some consecutive integer value of $k_{r}$, the reslult of $\beta(k, 1)$ will be a square number. With the increase in $k_{r}$ values in this zone, the number of consecutive integer values of $k_{r}$ are increased accordingly. Therefore it is only necessary to do a $\beta(k, 1)$ test for one value of $k_{r}$ 
based on the theorem(4). In general case, for an odd number as $F$, it is better to do the $\beta-S$ sieve as follows:

\begin{tabular}{|c|c|c|c|c|}
\hline \multicolumn{5}{|c|}{$k=1, \quad \Delta_{1-1} \Rightarrow k_{r}=\frac{y}{y} \rightarrow 4 k F \rightarrow S_{\text {e or o }}^{b_{1}}, S_{d_{1-1}}, \quad S_{u_{1-1}}$} \\
\hline$D$ & $S$ & $E_{N_{1}}$ & $E_{N_{2}}$ & \\
\hline \multicolumn{5}{|l|}{$\overline{D_{1}}$} \\
\hline \multicolumn{5}{|l|}{$D_{2}$} \\
\hline ............ & 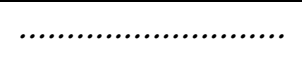 & 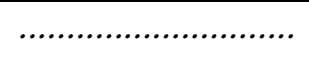 & .................................. & , \\
\hline \multicolumn{5}{|c|}{$k=2, \quad \Delta_{2-1} \Rightarrow k_{r}=\frac{2 y}{y} \rightarrow 4 k F \rightarrow S_{\text {e or o }}^{b_{1}} \quad, S_{d_{2-1}}, \quad S_{u_{2-1}}$} \\
\hline $\bar{D}$ & $S$ & $E_{N_{1}}$ & $E_{N_{2}}$ & \\
\hline \multicolumn{5}{|l|}{$D_{2-1}$} \\
\hline \multicolumn{5}{|l|}{$D_{2-2}$} \\
\hline ........... & . & ............................ & 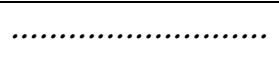 & 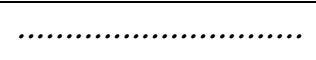 \\
\hline
\end{tabular}

Example3: By selecting $y=2 a n d ~ k=2$ prove that $F=9,640,669$ is a composite number.

Answer:

$$
\Delta_{2-1}
$$

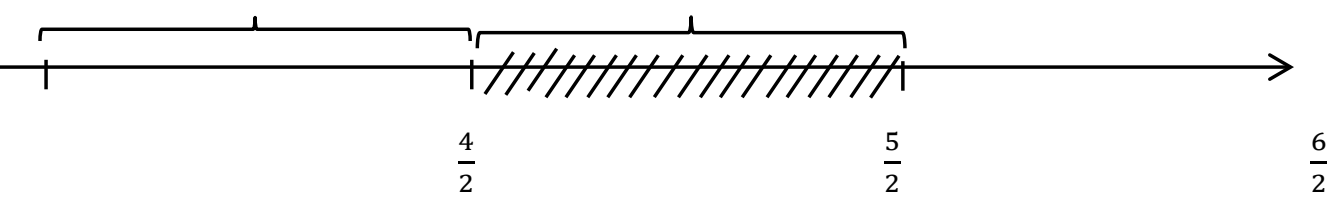

$, S_{d_{2-1}}=17,564 \quad, \quad S_{u_{2-1}}=\left\lceil 9 \sqrt{\frac{2 F}{5}}\right\rceil=17,673 \quad 4 k F=4 \times 4 \times 2 \times F=308,501,408$

We can see, when $S=17,626$ then $\beta$ is a prefect square number, since we have

$\beta=S_{r}^{2}-4 k F=(17,622)^{2}-308,501,608=1426^{2}=t^{2}$

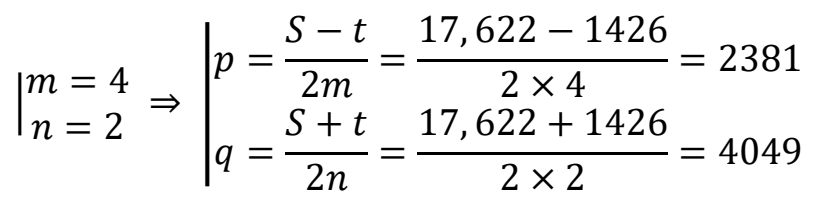

The important benefits of $\beta-S$ sieve is that we can easily select a certain sieve zone within any distance from $k_{r}$ values (from 1 to $F$ ). This is particularly very important for some values of $k_{r}$ since it includes a large part of the interval containing the $p$ values.

Example4: By the $\beta-S$ sieve show that the number $F=34,873,477$ in the sieve zone of $\Delta_{3-1}$ has an response point. 
Answer

$k=3 \quad, \quad 12 F=418,481,724, S_{d_{3-1}}=20,456, S_{u_{3-1}}=20,668$

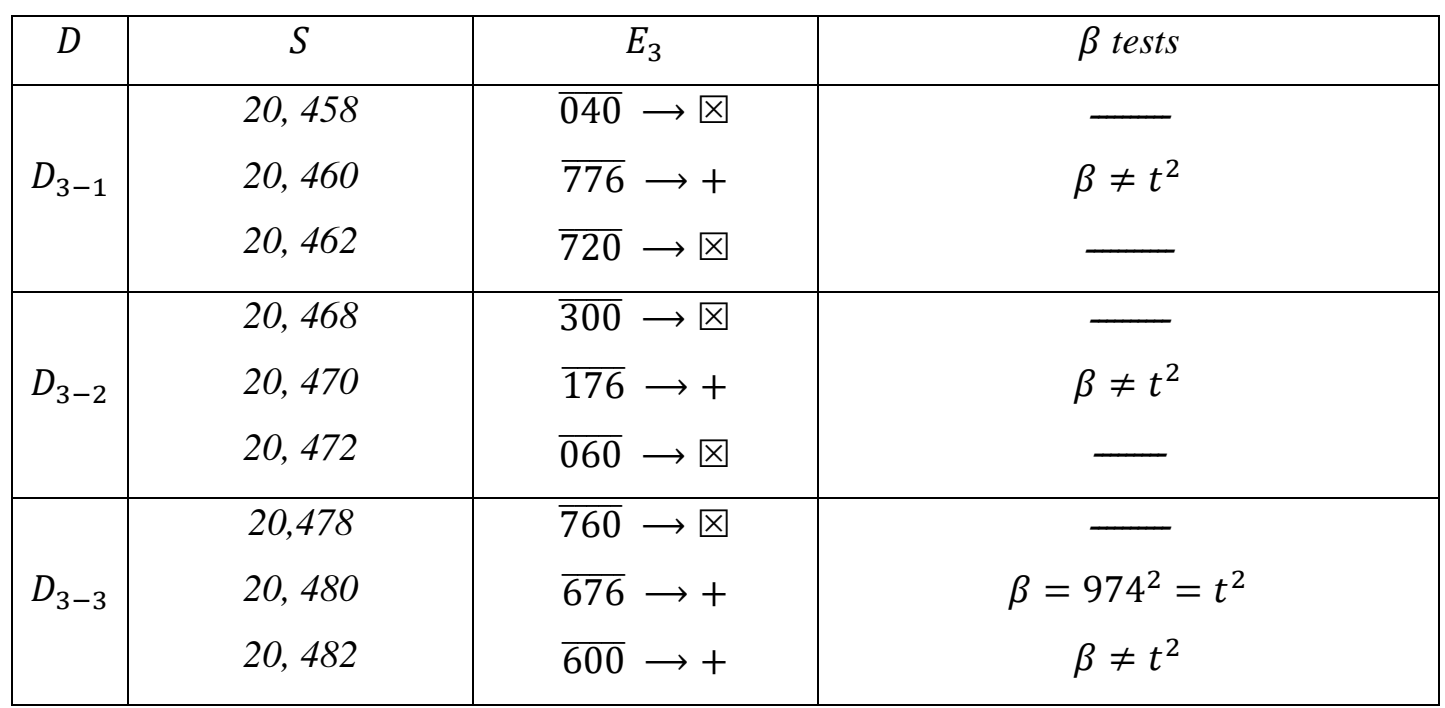

In order to utilize the $\beta-S$ sieve in the zone of $S=0$, it will only suffice to use the integer values of $k_{r}$, located in this zone, as the $k$ in $\beta(k, 1)$ tests.

If the value of $\sqrt[3]{\frac{F}{256}}$ is represented by $I$, therefore in the zone of $S=0$, from relations $\delta=4 \sqrt[4]{\frac{k_{r}^{3}}{F}}$ and $k_{r(n)}=\sqrt[3]{\left(\frac{n}{4}\right)^{4} F}$, we will have $k_{r(n)}=n \sqrt[3]{n} I$ then we can conclud that $\delta=N=n$.

In a way that $N$ represents the number of $k$ values for one value of $k_{r}$ in order to have the same result for the $\beta(k, 1)$ tests. Therefore it is suggested that the distance from I to F can be divided as follows:

$$
k_{r}=I \quad k_{r}=2 \sqrt[3]{2} I \quad k_{r}=3 \sqrt[3]{3} I \quad k_{r}=4 \sqrt[3]{4} I \quad k_{r}=5 \sqrt[3]{5} I \quad \ldots \ldots k_{r}=F
$$

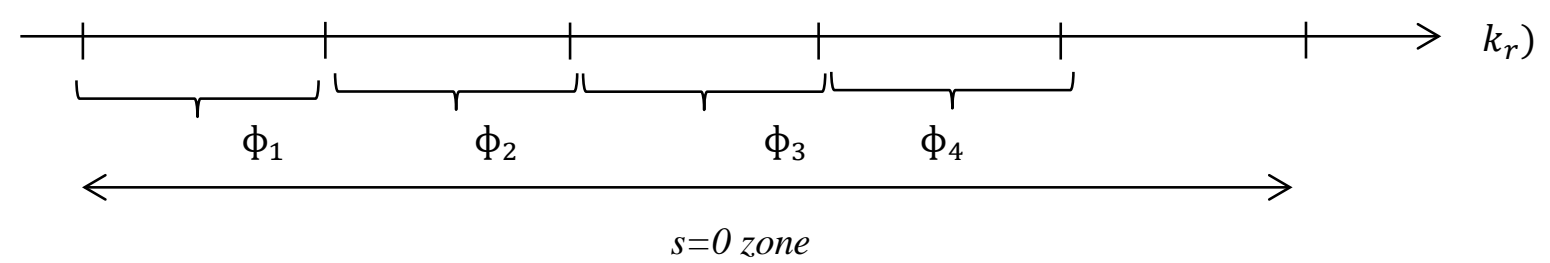

In an arbitrary interval as $\phi_{n}$, some values of $k$ must be used in $\beta(k, 1)$ test which are obtained from the $k_{n}+n i$ in a way that $k_{n}=\lceil n \sqrt[3]{n} I\rceil$ is the minimum value of $k$ and $k_{n+1}=\lfloor(n+1) \sqrt{n+1} I\rfloor$ is the maximum value of $k$.
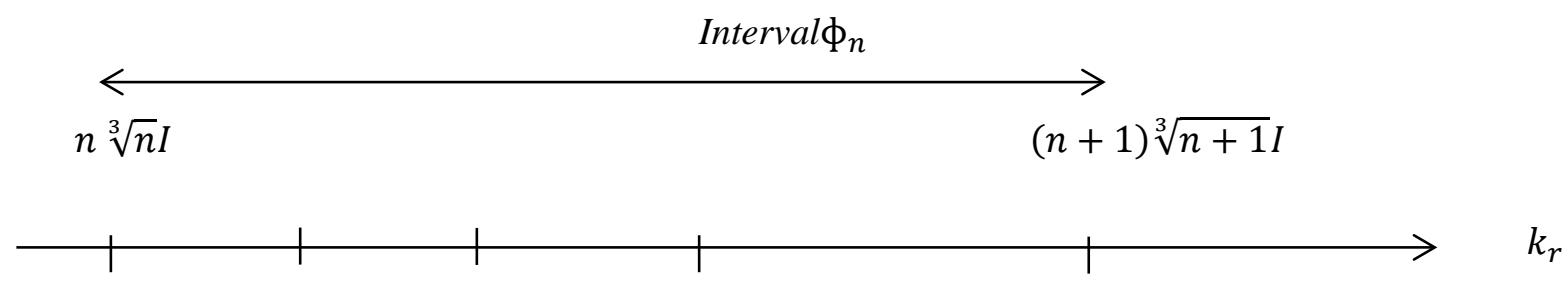


$$
k_{n}=[n \sqrt[3]{n} I\rceil \quad k_{n}+n \quad k_{n}+2 n \quad k_{n}+3 n \quad \ldots \quad\lfloor(n+1) \sqrt[3]{n+1} I\rfloor=k_{n+1}
$$

We represent the numbers of $k$ that can be chose for $\beta(k, 1)$ tests in $\phi_{n}$ interval by $N_{n}$, that is:

$N_{n}=\left\lceil\frac{(n+1) \sqrt[3]{n+1} I-n \sqrt[3]{n} I}{n}\right\rceil=\left\lceil\frac{L_{n}}{n}\right\rceil$

It is observed that by increase in $n$, the length of the $\phi_{n}$ intervals, i.e. $L_{n}$, will also increase. In other words we have:

$$
\begin{aligned}
& (2 \sqrt[3]{2} I-I)<(3 \sqrt[3]{3} I-2 \sqrt[3]{2} I)<(4 \sqrt[3]{4} I<3 \sqrt[3]{3} I)<\cdots \Rightarrow L_{1}<L_{2}<L_{3}<\cdots<L_{m}<L_{m+1}<\cdots \\
\Rightarrow & N_{1} \geq N_{2} \geq N_{3} \geq \cdots \geq 1
\end{aligned}
$$

In order to calculate the maximum value of $n$ in $S=0$ zone, we will have:

$(n+1) \sqrt[3]{n+1} I=\sqrt[3]{(n+1)^{4}} \times \sqrt[3]{\frac{F}{256}}=F \Rightarrow n_{\max }=|4 \sqrt{F}|-1$

Therefore if we want to have $N=1$, from differential calculus, we have:

$(n+1) \sqrt[3]{n+1} I-n \sqrt[3]{n} I=\left(\sqrt[3]{(n+1)^{4}}-\sqrt[3]{n^{4}}\right) I \approx\left(\frac{4}{3} \sqrt[3]{n}\right) I<n \Rightarrow n \geq\left\lceil\frac{\sqrt{3 F}}{18}\right\rceil$

As an example, for the number $F=631,523$ we will have $\left\lceil\frac{\sqrt{3 F}}{18}\right\rceil=77$, therefore If $n=77$ then we should have:

$L_{77}=78 \sqrt[3]{78} I-77 \sqrt[3]{77} I=76.737 \Rightarrow N_{77}=\left\lceil\frac{L_{77}}{77}\right\rceil=1$

It means that for values of $n$ which are greater than 77 , it will be suffice to select an integer number for the $\beta$ test in each $\phi$ intrval.

For example, in $S=0$ zone, for the number $=67,813$, we have:
$25 \sqrt[3]{25} I$
$36 \sqrt[3]{36} I$
$1040 \sqrt[3]{1040} I$
F

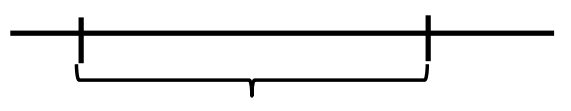

$\approx 476$

$\approx 492$

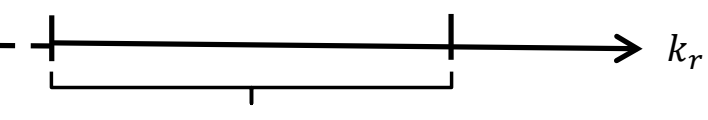

$\approx 67,435$

67,812

It is observed that:

$\left\{\begin{array}{c}L_{25}=16 \\ N_{25}=1\end{array} \quad, \ldots \ldots \ldots \ldots \ldots \ldots,\left\{\begin{array}{c}L_{1040}=378 \\ N_{1040}=1\end{array}\right.\right.$

From differential calculus, we know that: 
$f(n+\Delta n)-f(n) \approx f^{\prime}(n) \Delta n, f(n)=\sqrt[3]{n^{4}}, f^{\prime}(n)=\frac{4}{3} \sqrt[3]{n^{4}}$

$\Delta n=1 \Rightarrow \sqrt[3]{(n+1)^{4}}-\sqrt[3]{n^{4}} \approx \frac{4}{3} \sqrt[3]{n^{4}} \approx \frac{n N}{I}=n N \sqrt[3]{\frac{256}{F}} \Rightarrow n=\left\lceil\frac{1}{6 N} \sqrt{\frac{F}{3 N}}\right\rceil$

$\Rightarrow N=\left\lceil\sqrt[3]{\frac{F}{108 n^{2}}}\right\rceil \Rightarrow N_{\max }=\left\lceil\sqrt[3]{\frac{F}{108}}\right\rceil$

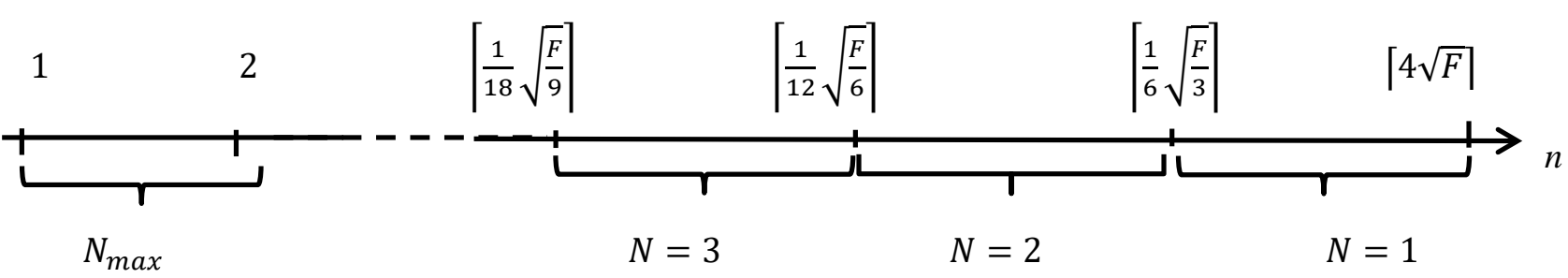

For any $\beta(k, 1)$ value in $\phi_{n}$ intervals, we can use $E_{N}$ tests.

\section{Acknowledgments}

The author would like to express his sincere gratitude to Dr. Amir Jafari, associated professor of Sharif University, for his guidance in all steps. I also want to thank my wife, Fariba, and my son Alireza, for their kind encouragement in all conditions.

\section{References:}

1.D.M. Burton, “Elementary Number Theory”, Mc Graw Hill Companies, 2007.

2.R. Cranal, C. Pomerance, “Prime Numbers”, Springer, 2005.

3.D. Wells, "Prime Numbers”, Joun Wiley \& Sons, 2005.

4.P. Hackman, “Elementary Number Theory”, HHH. Production, 2009.

5.T. Koshy, “Elementary Number Theory with Application”, Elsevier, 2007.

6.K.C. Chowdhury, "A First course in Number theory”, Asian Books Private Limited, 2007.

7.W. Narkiewicz, “The Development of Prime Number theory”, Spring, 2000.

8.H.M. Stark, "An Introduction to Number theory”, MIT Press, 1987.

9.A. Baker, “A Comprehensive Course in Number theory”, Cambridge University Press, 2012.

10.K.H. Rosen, "Elementary Number theory”, Pearson Adisson Wisely, 2005.

11.J.J. Tattersall, “Elementary Number Theory”, Cambridge University Press, 2005.

12.G.A.Jones, M. Jones, “Elementary Number Theory”, Springer, 2005.

13.W. Sierpinsky, “Elementary Theory of Numbers”, PWN-Polish Scientific Publishers, 1991. 
14.M.B. Nathanson. "Elementary Methods in Number Theory”, Springer, 2000.

15.O. Ore, "Number Theory and Its History”, Mc Graw Hill Companies, 1948. 